\title{
On the continuous equilibria of affiliated-value, all- pay auctions with private budget constraints
}

\section{Citation}

Kotowski, Maciej H., and Fei Li. 2014. "On the continuous equilibria of affiliated-value, allpay auctions with private budget constraints." Games and Economic Behavior (February). doi:10.1016/j.geb.2014.01.014. http://dx.doi.org/10.1016/j.geb.2014.01.014.

\section{Published Version}

doi:10.1016/j.geb.2014.01.014

\section{Permanent link}

http://nrs.harvard.edu/urn-3:HUL.InstRepos:11718166

\section{Terms of Use}

This article was downloaded from Harvard University's DASH repository, and is made available under the terms and conditions applicable to Open Access Policy Articles, as set forth at http:// nrs.harvard.edu/urn-3:HUL.InstRepos:dash.current.terms-of-use\#OAP

\section{Share Your Story}

The Harvard community has made this article openly available.

Please share how this access benefits you. Submit a story.

Accessibility 


\title{
On the Continuous Equilibria of Affiliated-Value, All-Pay Auctions with Private Budget Constraints*
}

\author{
Maciej H. Kotowski ${ }^{\dagger} \quad$ Fei $\mathrm{Li}^{\ddagger}$
}

January 10, 2013

\begin{abstract}
We consider all-pay auctions in the presence of interdependent, affiliated valuations and private budget constraints. For the sealed-bid, all-pay auction we characterize a symmetric equilibrium in continuous strategies for the case of $N$ bidders. Budget constraints encourage more aggressive bidding among participants with large endowments and intermediate valuations. We extend our results to the war of attrition where we show that budget constraints lead to a uniform amplification of equilibrium bids among bidders with sufficient endowments. An example shows that with both interdependent valuations and private budget constraints, a revenue ranking between the two auction formats is generally not possible. Equilibria with discontinuous bidding strategies are discussed.
\end{abstract}

Keywords: All-Pay Auction, War of Attrition, Budget Constraints, Common Values, Private Values, Affiliation, Contests JEL: D44

*This paper generalizes our previous working paper, "All-Pay Auctions with Budget Constraints: The Two-Bidder Case." That paper merged the independent and simultaneous work of Kotowski (2010) and Li (2010). We are grateful to the editor and the referees for helpful comments.

†John F. Kennedy School of Government, Harvard University, 79 JFK Street, Cambridge MA 02138. E-mail: <maciej_kotowski@hks.harvard.edu>.

$\ddagger$ (Corresponding Author) Department of Economics, 107 Gardner Hall, CB 3305, University of North Carolina, Chapel Hill NC 27599. E-mail: <lifei@email.unc.edu>. 
Suppose firms are lobbying for a lucrative government contract. The contract's value to each firm has an idiosyncratic component since the firms likely have different operating costs. But also, each firm has a privately-known limit on how much it is able to spend on the lobbying game. Perhaps the management of one firm is approving of restaurant meals with officials but expenditures or bribes beyond some threshold are morally too much to stomach. A competitor, in contrast, may be less hampered in its lobbying strategy. How does the lobbying game unfold when competitors differ in their valuation for the prize and in their ability or capacity to compete for it? Would some firms spend more on lobbying believing that their competitors have to navigate within some private and binding constraints on actions?

In this essay we consider a class of situations not unlike the above lobbying contest by analyzing all-pay auctions. In a (first-price) all-pay auction, the highest bidder is the winner of the item for sale; however, all bidders incur a payment equal to their bid. As a stylized model of a lobbying contest, the all-pay auction has an established tradition in political economy (Hillman and Riley, 1989; Baye et al., 1993). ${ }^{1}$

Despite the frequent application of the all-pay auction to models of contests, most analyses fail to capture the exogenous, but private, limits on actions that are commonly encountered. In practice all participants face a budget constraint, a hard deadline, or a maximum level of feasible effort. Ignoring these constraints has hitherto been a helpful modeling simplification. We argue, however, that this simplification has masked much of the nuance embedded in the situation. Our analysis introduces private constraints into the all-pay auction with interdependent and affiliated valuations. We identify sufficient conditions for the existence of an equilibrium in continuous strategies. We also provide an extension of our model to the (static) war of attrition to show the broader applicability of our analysis.

Although our model is phrased in the language of auctions (players are called "bidders," etc.), it applies to any situation where resources are irreversibly expended in pursuit of a goal or a prize. The goal or prize can have a value that has both private and common components. Our model accommodates both cases. The private constraints on bids or effort that we introduce are often natural elements of the situation. The constraints may be financial, physical, or amalgams of many component factors.

As one specific example of the range of applications, consider college admissions. Hickman

\footnotetext{
${ }^{1}$ We focus only on auction mechanisms where a bidder placing the highest bid is the winner. Probabilistic contests in the sense of Tullock (1980) are beyond this paper's scope. When we refer to "contests" we have in mind the special case that we are analyzing. Konrad (2009) provides a survey of the literature on contests more broadly, and includes a discussion of all-pay auctions.
} 
(2011) employs a version of the all-pay auction to model students competing for places in a college. Those who "bid" the most, by exerting irreversible effort, are more likely to gain a scarce spot in the school. It is clear that the benefit from a college degree varies across students due to personal preferences and characteristics. Hence, different students value college attendance to varying degrees. It is also natural to assume that idiosyncratic shocks, such as health status, family background, parental savvy, or school location, place an exogenous, heterogenous, and private cap on the effort that a particular student can exert in the college admissions game. ${ }^{2}$

As another example, consider a patent race between competing firms. Such competition is naturally modeled as either an all-pay auction or as a war of attrition (Leininger, 1991). The expected value of the invention and the budget available to a company's research division will determine the effort devoted to the race. Information asymmetries or agency concerns can create a wedge between the available budget and the research division's assessment of the project's value. Moreover, each firm likely faces a hard, short-run physical resource constraint. This constraint will cap its feasible effort level. The interaction between expected rewards and heterogenous resource constraints will shape how firms engage in this competition.

While we are motivated by the range of social and economic situations that all-pay auctions can model, our study also fills a gap in the growing literature on auctions with private budget constraints. Our analysis builds directly on the work of Krishna and Morgan (1997) who study the all-pay auction and the war of attrition with interdependent and affiliated valuations. Their analysis extends the general symmetric model of Milgrom and Weber (1982) to these more unusual auction procedures. To this setting we introduce private budget constraints distributed continuously on an interval. Our environment parallels the setting of Fang and Parreiras $(2002,2003)$ and Kotowski (2013) who study the second-price and the first-price auction with private budget constraints, respectively. These latter studies build directly on Che and Gale (1998b), which is the seminal paper in the literature on standard auctions incorporating private budget constraints. Che and Gale (1996) develop a simple model of an all-pay auction with private budget constraints where the item for purchase has a common and perfectly known value. That model is a limiting case of our environment. Finally, there is also a literature on publicly-known spending or bidding caps in all-pay auctions, or in contests more generally (Che and Gale, 1998a; Gavious et al., 2002).

\footnotetext{
${ }^{2}$ For a discussion concerning the strategic aspects of college applications and admissions see Avery et al. (2004).
} 
In our study, the spending or bidding limit of each bidder is private information.

In light of this literature, our study contributes along several dimensions. First, by focusing on all-pay mechanisms we put under scrutiny an important allocation mechanism in resource-constrained environments. Many authors examining optimal auctions with budgetconstrained participants have resorted to mechanisms that feature "all-pay" payment schemes (Laffont and Robert, 1996; Maskin, 2000; Pai and Vohra, Forthcoming). Our analysis therefore complements this literature, but we do not attempt the mechanism design exercise here.

Second, our model is set in a more general environment than traditionally employed when analyzing auctions with private budget constraints. Hence, we are able to identify additional features of the environment that affect the existence of a well-behaved and (relatively) tractable equilibrium. Previous studies lodged in the affiliated and interdependent-value paradigm, such as Fang and Parreiras (2002) and Kotowski (2013), have focused on the twobidder case. While some of the intuition from the two-bidder case is relevant generally, the case of two bidders masks many caveats. For example, in the all-pay auction we document how changes in the number of bidders alone directly affect the existence of an equilibrium within the class of strategies traditionally considered by this literature. This observation may be particularly valuable to future empirical analyses as it may be difficult to exploit a variation in the number of bidders to aid in model identification (Athey and Haile, 2007).

The equilibria that we construct in the all-pay auction and in the war of attrition are in monotone, continuous strategies. As discussed by Araujo et al. (2008), non-monotone equilibria often feature in multi-dimensional auction environments. ${ }^{3}$ In our setting, bidders have two dimensions of private information - a value-signal and a budget constraint - and interdependent valuations. Therefore the issues they address are related to our analysis. ${ }^{4} \mathrm{We}$ view our focus on monotone equilibria in continuous strategies as a pragmatic but reasonable choice. Although monotonicity is not a necessary condition to leverage the differential approach when characterizing equilibrium bidding (Araujo et al., 2008), it greatly simplifies our argument. To construct our equilibrium, we follow Che and Gale (1998b) by focusing on a specific class of bidding functions (explained below). In doing do, we effectively reparameterize our multi-dimensional problem into a simpler one-dimensional setting. The analysis of Araujo et al. (2008) shows that a transformation of the type-space is often a key step in analyzing multidimensional auction models. ${ }^{5}$ Our restriction notwithstanding, we

\footnotetext{
${ }^{3}$ See also Zheng (2001).

${ }^{4}$ Araujo et al. (2008) also propose an interesting application of the all-pay auction as a tie-breaking device in more complex auction-like games. Except for the analysis of Section 4, ties do not occur in our model.

${ }^{5}$ Che and Gale (2006) also employ a transformation of bidders' types to facilitate revenue comparisons in
} 
believe that the set of cases covered is rich and it offers insights that would carry over to a discontinuous equilibrium as well. Undoubtably, continuous equilibria would receive the bulk of attention in applications due to their relative tractability.

The remainder of the paper is organized as follows. Section 1 introduces the environment and section 2 studies the symmetric equilibrium in the all-pay auction. We then consider the equilibrium's comparative static properties with focus on changes in the distribution of budgets, changes in the number of bidders, and changes in the public information surrounding the contest. Section 3 considers this model's second-price analogue, the (static) war of attrition. We explore the symmetric equilibria of this model and we discuss the scope for a revenue ranking between the auction procedures examined in this study. We conclude with a brief consideration of equilibria in discontinuous strategies in the all-pay auction. Here we employ a stylized model to highlight features that we believe are economically salient in situations where bidders are budget constrained. ${ }^{6}$ Proofs and supporting lemmas are in the appendix. An online appendix collects additional results, extensions, and some technical arguments.

\section{The Environment}

Consider an auction where one good (or prize) is available. Let $\mathcal{N}=\{1, \ldots, N\}$ be the set of bidders. Each bidder $i \in \mathcal{N}$ has a two-dimensional private type, $\left(s_{i}, w_{i}\right) \in[0,1] \times[\underline{w}, \bar{w}]$. Suppose $0<\underline{w}<\bar{w} \cdot{ }^{7}$ First we describe the two dimensions of a bidder's type. Subsequently we introduce assumptions concerning the distribution of types and their statistical properties.

A bidder's realized value-signal, $s_{i}$, is her private information about the item for purchase. For example, in a patent race it would be an estimate of the invention's value. In a political lobbying contest, it may correspond to an assessment of the proposed legislation's consequences. Let $\mathbf{s}=\left(s_{1}, \ldots, s_{N}\right)$ be a profile of realized value-signals. ${ }^{8}$ We use capital letters - such as $S_{i}$ - to refer to signals as random variables.

A bidder's realized budget, $w_{i}$, is a bound above which she cannot bid. We consider a budget to be a hard constraint on expenditures. A budget may correspond to a bidder's cash holdings, her credit limit, or some other private limit on actions. Such limits may be

auctions.

${ }^{6}$ Kotowski (2013) discusses a similar model in application to the first-price auction.

${ }^{7}$ The case of $\underline{w}=0$ is addressed in the online appendix and is qualitatively similar to our main analysis.

${ }^{8}$ We use standard notation and shorthand: $\mathbf{s}_{-i}=\left(s_{1}, \ldots, s_{i-1}, s_{i+1}, \ldots, s_{N}\right), \mathbf{s}=\left(s_{i}, \mathbf{s}_{-i}\right)$, etc. 
financial, physical, or psychological, depending on the application of interest. ${ }^{9}$

Bidder $i$ 's valuation for the item can be described by a random variable: $V_{i}=u\left(S_{i}, S_{-i}\right)$. We assume that $u:[0,1] \times[0,1]^{N-1} \rightarrow[0,1]$ is strictly increasing in the first argument and non-decreasing and permutation-symmetric in the last $N-1$ arguments. As standard, we suppose $u$ is continuously differentiable. It is normalized such that $u(0, \ldots, 0)=0$ and $u(1, \ldots, 1)=1$. We assume that bidders are risk neutral.

While a player's realized type $\left(s_{i}, w_{i}\right)$ is private information, we assume that the distribution of types and the auction's ambient environment is common knowledge. Two assumptions concerning the distribution of bidders' types define our environment and we maintain them throughout our analysis. The first assumption concerns the distribution of value-signals while the second concerns the distribution of budgets. Subsequent assumptions, which are specific to the auction format considered, impose additional structure on our model.

Assumption A-1. Value-signals have a continuous, strictly positive joint density, $h:[0,1]^{N} \rightarrow$ $\mathbb{R}_{++}$. Moreover, $h\left(s_{1}, \ldots, s_{N}\right)$ is invariant to permutations of $\left(s_{1}, \ldots, s_{N}\right)$ and log-supermodular. ${ }^{10}$

Assumption A-1 means that value-signals are "affiliated." Affiliation is a standard assumption introduced to the auction literature by Milgrom and Weber (1982). It amounts to a special form of positive correlation that is amenable to the requisite formal arguments employed within most auction models. Independent signals are affiliated. A density exhibiting strict affiliation is, for example, $h\left(s_{1}, s_{2}\right)=\frac{4}{5}\left(1+s_{1} s_{2}\right)$. Although affiliation is a work-horse assumption in the auction literature, it is a restrictive statistical property (de Castro, 2010).

Concerning the distribution of players' budgets, we require budgets to be determined independently of value-signals and to be identically distributed.

Assumption A-2. Each bidder's budget is independently and identically distributed according to the differentiable cumulative distribution function $G(w)$. Its density, $G^{\prime}(w) \equiv g(w)$, is strictly positive for all $[\underline{w}, \bar{w}]$ and continuous. ${ }^{11}$

While the independence condition is strong, without it the model is not tractable. It is standard in studies of auctions with budget constraints when there is some affiliation in

\footnotetext{
${ }^{9}$ We focus on hard budget constraints. Some studies, such as Zheng (2001) or Che and Gale (2006), examine "softer" constraints on bidding, such as convex bid-financing costs. Extending our analysis in this direction is a possible avenue for further research.

${ }^{10} h(\cdot)$ is $\log$-supermodular when it satisfies the following property: For any $\mathbf{s}$ and $\mathbf{s}^{\prime}, h(\mathbf{s}) h\left(\mathbf{s}^{\prime}\right) \leq h(\mathbf{s} \vee$ $\left.\mathbf{s}^{\prime}\right) h\left(\mathbf{s} \wedge \mathbf{s}^{\prime}\right)$ where $\mathbf{s} \vee \mathbf{s}^{\prime}\left(\mathbf{s} \wedge \mathbf{s}^{\prime}\right)$ is the component-wise maximum (minimum) of $\mathbf{s}$ and $\mathbf{s}^{\prime}$.

${ }^{11}$ It is understood that $w \leq \underline{w} \Longrightarrow G(w)=0$ and $w>\bar{w} \Longrightarrow G(w)=1$.
} 
players' value-signals. Our model naturally accommodates the case of $\bar{w}=\infty$, and occasionally to present such examples, but for brevity we phrase our main discussion assuming $\bar{w}<\infty$.

A bidding strategy for bidder $i$ is a (measurable) function $\beta_{i}:[0,1] \times[\underline{w}, \bar{w}] \rightarrow \mathbb{R}_{+}$. Throughout, we adopt Bayesian-Nash equilibrium as our solution concept. An equilibrium is symmetric if all bidders follow the same bidding strategy. We focus on symmetric equilibria and we henceforth suppress player subscripts in our notation whenever possible.

Above we noted that we focus on a specific class of equilibrium strategies. To elaborate, our analysis seeks to identify a symmetric equilibrium where all bidders follow a strategy of the form

$$
\beta(s, w)=\min \{b(s), w\}
$$

where $b(s)$ is strictly increasing, continuous, and piecewise differentiable. We say that a bidding strategy with these properties is a canonical bidding strategy. Our focus on equilibria meeting these criteria is consistent with previous studies of auctions with private budget constraints. Che and Gale (1998b), Fang and Parreiras (2002, 2003), and Kotowski (2013) examine equilibria that reside in this class of strategies.

Before presenting our analysis, we consolidate some notational miscellany. Suppressing the " $i$ " subscript, we let $S$ be the value-signal observed by a bidder and we relabel the valuesignals of the other bidders as $Y_{1}, \ldots, Y_{N-1}{ }^{12}$ Let $\bar{Y}_{k}=\max \left(Y_{1}, \ldots, Y_{k}\right)$ and define $f_{k}(y \mid s)$ to be the density of $\bar{Y}_{k} \mid S=s$. For $k \geq 1$, we define the following terms:

$$
\begin{aligned}
v_{k}(s, y) & =\mathbb{E}\left[u\left(s, Y_{1}, \ldots, Y_{N-1}\right) \mid S=s, \bar{Y}_{k}=y\right] \\
z_{k}(x \mid s) & =\int_{0}^{x} v_{k}(s, y) f_{k}(y \mid s) d y
\end{aligned}
$$

For $k=0$, we adopt the convention that $v_{0}(s, y)=\mathbb{E}\left[u\left(s, Y_{1}, \ldots, Y_{N-1}\right) \mid S=s\right]$ and $z_{0}(x \mid s)=$ $\mathbb{E}\left[u\left(s, Y_{1}, \ldots, Y_{N-1}\right) \mid S=s\right]$. Hence $v_{0}(s, y)$ is constant in $y$ and $z_{0}(x \mid s)$ is constant in $x$.

Finally, we will frequently need to manipulate binomial terms to account for the likelihood that a specific number of bidders has a budget less than some value. In these cases we will employ the shorthand

$$
\gamma_{k}(b)=\left(\begin{array}{c}
N-1 \\
k
\end{array}\right) G(b)^{N-1-k}(1-G(b))^{k} .
$$

\footnotetext{
${ }^{12}$ We only relabel the value signals. We do not reorder them as some authors do.
} 
$\gamma_{k}(b)$ is the probability that exactly $N-1-k(k)$ bidders out of $N-1$ have a budget less (greater) than $b$.

\section{The All-Pay Auction}

The rules of the all-pay auction are well-known. Each bidder $i$ simultaneously submits a bid $b_{i}$. The highest bidder is deemed the auction's winner. (Ties among high bidders are resolved by a uniform randomization.) If bidder $i$ is declared the auction's winner, her payoff given the realized signal profile $\mathbf{s}=\left(s_{i}, \mathbf{s}_{-i}\right)$ is $u\left(s_{i}, \mathbf{s}_{-i}\right)-b_{i}$; otherwise, it is $-b_{i}$. We assume that a submitted bid must be feasible give a bidder's budget constraint. Thus, a bidder of type $\left(s_{i}, w_{i}\right)$ may only bid less than $w_{i}$.

To motivate the sufficient conditions for equilibrium existence that we will propose below, we begin with an heuristic discussion that is suggestive of their origin. Suppose that there is a symmetric equilibrium in the all-pay auction where each bidder adopts the canonical strategy $\beta(s, w)=\min \{b(s), w\}$. Suppose a bidder places the bid $b(x)$ for some $x \in[0,1]$. This bid will defeat two categories of opponents assuming all other bidders are following the strategy $\beta(s, w)$. First it defeats all opponents who have a value-signal $s<x$. Second, it defeats all opponents who have a budget $w<b(x) .{ }^{13}$ Noting this fact, we can use (3) and (4) to write the expected payoff of bidder $i$ when she bids $b(x)$ as

$$
U_{i}(b(x) \mid s, w)=\sum_{k=0}^{N-1} \gamma_{k}(b(x)) z_{k}(x \mid s)-b(x)
$$

We outline in greater detail the derivation of (5) in the appendix. The binomial terms account for the combinations of opponents who are defeated by $b(x)$ due to having a low value-signal or a low budget. When values are interdependent, defeating an opponent because she has a low value-signal or a low budget carry distinct implications and our accounting in (5) acknowledges this caveat. The final term in (5) is the bidder's payment which she makes irrespective of the auction's outcome.

If in equilibrium we observe a bidder of type $(s, w)$ bid $b(s)<w$, then the bid must satisfy a local, first-order optimality condition. Specifically, assuming appropriate differentiability,

$$
\left.\frac{d}{d x} U_{i}(b(x) \mid s, w)\right|_{x=s}=0 .
$$

\footnotetext{
${ }^{13}$ Given the maintained assumptions, ties are probability-zero events.
} 
Adopting the notation

$$
z_{k}^{\prime}(x \mid s) \equiv \frac{\partial}{\partial x} z_{k}(x \mid s)= \begin{cases}0 & \text { if } k=0 \\ v_{k}(s \mid x) f_{k}(x \mid s) & \text { if } k \neq 0\end{cases}
$$

we can evaluate (6) and rearrange terms to arrive at

$$
b^{\prime}(s)=\frac{\sum_{k=0}^{N-1} \gamma_{k}(b(s)) z_{k}^{\prime}(s \mid s)}{1-\sum_{k=0}^{N-1} \gamma_{k}^{\prime}(b(s)) z_{k}(s \mid s)}
$$

If there exists a symmetric equilibrium in canonical strategies, the differential equation in (7) represents a first guess concerning the nature of $b(s)$. Our subsequent discussion identifies conditions ensuring that (7) has a solution that is consistent with a symmetric equilibrium.

Two initial observations regarding (7) are immediate. First, when $b(s)<\underline{w}$ equation (7) reduces to

$$
b^{\prime}(s)=v_{N-1}(s, s) f_{N-1}(s \mid s) .
$$

This is the differential equation identified by Krishna and Morgan (1997) as defining the equilibrium bidding strategy in the all-pay auction absent budget constraints. In an environment satisfying their regularity conditions, in equilibrium a bidder with a value-signal of $s$ will bid

$$
\alpha(s)=\int_{0}^{s} v_{N-1}(y, y) f_{N-1}(y \mid y) d y
$$

Given the tight connection between (7) and (8), whatever sufficient conditions we propose ought to generalize those proposed by Krishna and Morgan (1997). An immediate corollary to this observation is that if budget constraints are "not relevant," our model reduces to their analysis. Thus, we henceforth assume that $\underline{w} \leq \bar{\alpha} \equiv \alpha(1)$. Otherwise, $\alpha(s)$ would be the equilibrium and our analysis would be trivial.

Second, when $b(s)>\underline{w},(7)$ does not reduce any further. Instead, the complex expression accounts for the changing marginal effectiveness of bidding. Slight bid increases not only defeat opponents with slightly higher valuations but they also defeat all opponents with sufficiently low budgets regardless of their valuation. This second effect serves to ameliorate the winner's curse when values are interdependent.

Regrettably the derivation of (7) was heuristic and we made many implicit assumptions along the way. Specifically, we need to ensure that the solution to (7) satisfying an appropriate boundary condition is strictly increasing (whenever less than $\bar{w}$ ). For example, if the 
denominator of $(7)$ is ever negative, then $b^{\prime}(s)<0$, contradicting our original working hypothesis that $b(s)$ is increasing. Furthermore, we must also ensure that first-order conditions are sufficient to pin-down a bidder's optimal bid, which in general may not be true.

To address the above concerns we introduce two additional assumptions. Speaking loosely and intuitively, the first assumption will limit the "degree of affiliation" among bidders" value-signals. The second assumption will place a restriction on the joint distribution of value-signals and budgets. Both assumptions speak to the complicated interaction among the conflicting incentives faced by bidders in the all-pay auction.

The first assumption generalizes the sufficient condition proposed by Krishna and Morgan (1997) supporting $\alpha(s)$ as the equilibrium strategy in the all-pay auction without budget constraints.

Assumption A-3. Let $\phi(x, w \mid s)=\sum_{k=1}^{N-1} \gamma_{k}(w) v_{k}(s, x) f_{k}(x \mid s)$. For all $(x, w), \phi(x, w \mid \cdot):[0,1] \rightarrow$ $\mathbb{R}$ is non-decreasing. ${ }^{14}$

Remark 1. When $w \leq \underline{w}, \phi(x, w \mid s)=v_{N-1}(s, x) f_{N-1}(x \mid s)$. Hence, Assumption A-3 generalizes a sufficient condition for equilibrium existence identified by Krishna and Morgan (1997) in their model of the all-pay auction. In our notation, their condition states that $v_{N-1}(\cdot, x) f_{N-1}(x \mid \cdot):[0,1] \rightarrow \mathbb{R}$ is non-decreasing.

Intuitively, Assumption A-3 limits the degree of correlation among value-signals relative to the impact of a player's own value-signal on her valuation. The assumption always holds if signals are independent but it can hold in other cases as well. For example, it is satisfied when there are two bidders, $u\left(s_{i}, s_{j}\right)=\left(s_{i}+s_{j}\right) / 2$ and $h\left(s_{i}, s_{j}\right)=\frac{4}{5}\left(1+s_{i} s_{j}\right)$.

Whereas Assumption A-3 places a restriction on the correlation among value-signals, we additionally require an assumption structuring the joint distribution of value-signals and budgets. Assumption A-4 presents this restriction. We defer interpreting Assumption A-4 until after presenting our main result and an example illustrating the identified equilibrium. We define the value $\tilde{s}_{\alpha}$ as the unique solution to $\alpha\left(\tilde{s}_{\alpha}\right)=\underline{w}$.

Assumption A-4. Let $\xi(x, w \mid s)=1-\sum_{k=0}^{N-1} \gamma_{k}^{\prime}(w) z_{k}(x \mid s)$. Then the following conditions hold:

1. For every $s \geq \tilde{s}_{\alpha}$, there exists $w_{s}, \underline{w} \leq w_{s}<\bar{w}$, such that $w<w_{s} \Longrightarrow \xi(s, w \mid s)<0$ and $w>w_{s} \Longrightarrow \xi(s, w \mid s)>0$.

\footnotetext{
${ }^{14}$ Alternatively, we can write $\phi(x, w \mid s)=\sum_{k=0}^{N-1} \gamma_{k}(w) z_{k}^{\prime}(x \mid s)$. Both notations are useful for different steps in the analysis.
} 
2. There exists $\epsilon>0$ such that $s \in\left(\tilde{s}_{\alpha}-\epsilon, \tilde{s}_{\alpha}+\epsilon\right) \Longrightarrow \xi(s, \underline{w} \mid s)>0$.

3. When $x \geq \tilde{s}_{\alpha}, \xi(x, w \mid \cdot):[0,1] \rightarrow \mathbb{R}$ is non-increasing.

Although Assumption A-4 may appear to be a strictly technical statement, it has an economic interpretation that we discuss after introducing our main result.

Theorem 1. Suppose Assumptions A-1-A-4 are satisfied. There exists a symmetric equilibrium in continuous strategies in the all-pay auction with private budget constraints. In this equilibrium, all bidders follow the strategy $\beta(s, w)=\min \{b(s), w\}$ defined as follows:

- For all $s<\tilde{s}_{\alpha}, b(s)=\alpha(s)=\int_{0}^{s} v_{N-1}(y, y) f_{N-1}(y \mid y) d y$.

- For all $s \geq \tilde{s}_{\alpha}, b(s)$ is the strictly increasing solution of the differential equation

$$
b^{\prime}(s)=\frac{\sum_{k=0}^{N-1} \gamma_{k}(b(s)) z_{k}^{\prime}(s \mid s)}{1-\sum_{k=0}^{N-1} \gamma_{k}^{\prime}(b(s)) z_{k}(s \mid s)}
$$

satisfying the boundary condition $b\left(\tilde{s}_{\alpha}\right)=\underline{w}$.

Remark 2. Noting Theorem 1 it is clear that Assumption A-2 is stronger than strictly necessary. Theorem 1 requires $g(\cdot)$ to be smooth only in a "relevant range" of values so that (10) has a well-defined solution. We maintain the more-restrictive-than-necessary conditions of Assumption A-2 to unify or exposition of the all pay auction and the war of attrition.

Remark 3. If $u\left(\cdot, \mathbf{s}_{-i}\right) h\left(\mathbf{s}_{-i} \mid \cdot\right):[0,1] \rightarrow \mathbb{R}_{+}$is non-decreasing and absolutely continuous for each $\mathbf{s}_{-i}$, Assumptions A-3 and A-4(3) are satisfied. This condition may be simpler to verify in applications. It also allows for an alternative set of sufficient conditions supporting the existence of an equilibrium in the all-pay auction (Kotowski and Li, 2012).

The following example highlights several features of the all-pay auction equilibrium.

Example 1. Suppose $N=2, S_{i} \stackrel{\text { i.i.d. }}{\sim} U[0,1]$, and $W_{i} \stackrel{\text { i.i.d. }}{\sim} U\left[\frac{2}{25}, \frac{3}{4}\right]$. Let $u\left(s_{i}, s_{j}\right)=\left(s_{i}+s_{j}\right) / 2$. The symmetric equilibrium strategy is $\beta(s, w)=\min \{b(s), w\}$ where $b(s)$ is defined as follows:

- For $s<\tilde{s}_{\alpha}=2 / 5, b(s)=s^{2} / 2$.

- For $s \geq \tilde{s}_{\alpha}=2 / 5, b(s)$ is the solution to the differential equation

$$
b^{\prime}(s)=\frac{25(3-4 b(s)) s}{25 s(3 s-2)+42}
$$

satisfying the boundary condition $b\left(\frac{2}{5}\right)=\frac{2}{25}$. 


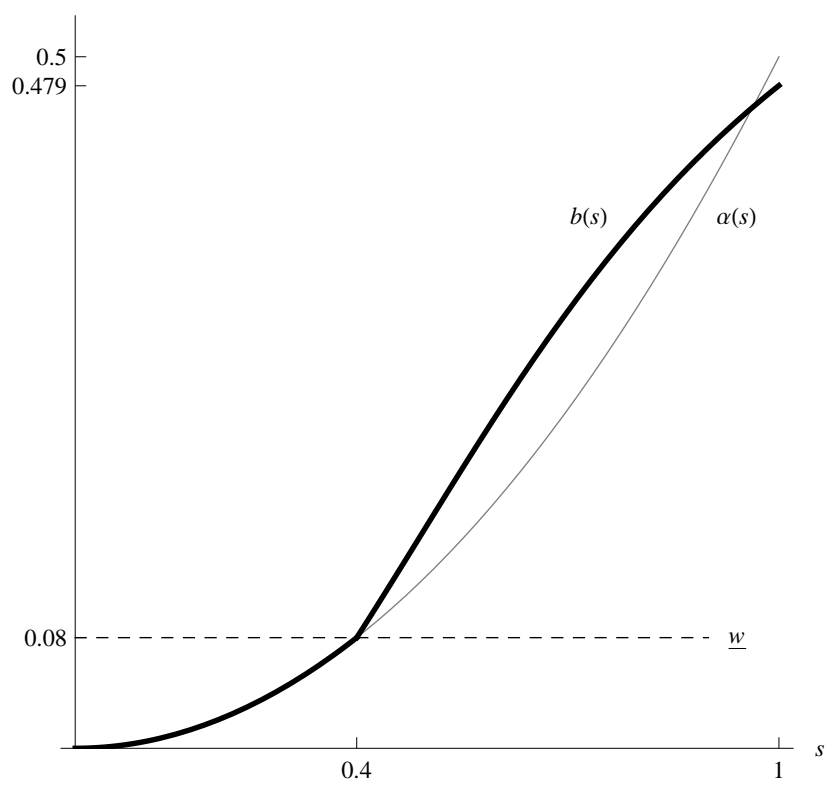

Figure 1: The functions $b(s)$ and $\alpha(s)$ from Example 1.

Figure 1 plots the functions $b(s)$ and $\alpha(s)=\frac{s^{2}}{2}$, which is the equilibrium strategy in this environment absent budget constraints. ${ }^{15}$ The introduction of budget constraints rendered $b(s)$ concave for $s>\tilde{s}_{\alpha}$ while $\alpha(s)$ is convex. Immediately to the right of $\tilde{s}_{\alpha}=2 / 5, b(s)>\alpha(s)$; therefore, some types of bidders with intermediate value-signals bid more following the introduction of budget constraints. Corollary 1 demonstrates that such a targeted amplification is a common feature of equilibrium bidding in the presence of budget constraints.

Corollary 1. Under the conditions of Theorem 1, $\lim _{s \rightarrow \tilde{s}_{\alpha}^{+}} b^{\prime}(s)>\lim _{s \rightarrow \tilde{s}_{\alpha}^{-}} b^{\prime}(s)$.

The encouragement of more aggressive bidding by bidders with relatively large budgets and intermediate valuations is due to a change in the marginal incentives that bidders experience in the presence of budget constraints. The prospect of defeating additional opponents who are budget-constrained increases the marginal return of a higher bid; therefore, some types of bidders respond to this incentive with more aggressive bidding.

\subsection{Discussion and Interpretation}

To interpret the sufficient conditions behind Theorem 1 it is useful to examine in detail the role of Assumption A-4. Assumption A-4(1) asserts that the function $\xi(s, \cdot \mid s)$ satisfies

\footnotetext{
${ }^{15}$ In all examples, plots of numerical solutions are obtained using the Runge-Kutta method.
} 
a single-crossing condition and $\xi(s, w \mid s)$ is strictly positive for $w$ sufficiently large. Thus, the assumption ensures that the righthand side of the differential equation (7) is eventually strictly positive. While this is Assumption A-4's technical role, it also has an economic interpretation.

Writing the condition $\xi(s, w \mid s)>0$ explicitly $^{16}$ gives

$$
g(w)(N-1)\left[\sum_{k=0}^{N-2}\left(\begin{array}{c}
N-2 \\
k
\end{array}\right) G(w)^{N-2-k}(1-G(w))^{k}\left(z_{k}(s \mid s)-z_{k+1}(s \mid s)\right)\right]<1
$$

To simplify further, suppose values are private and value-signals are independent draws from a common distribution with c.d.f. $H(s)$. Under these additional assumptions, we benefit from the simplification $z_{k}(s \mid s)-z_{k+1}(s \mid s)=u(s) H(s)^{k}(1-H(s))$ and we are able to rewrite $\xi(s, w \mid s)>0$ as

$$
\begin{aligned}
& g(w)(N-1) u(s)(1-H(s))(G(w)+H(s)-G(w) H(s))^{N-2}<1 \\
& \Longleftrightarrow u(s) \frac{d}{d w}[G(w)+H(s)-G(w) H(s)]^{N-1}<1
\end{aligned}
$$

The term $[G(w)+H(s)-G(w) H(s)]^{N-1}$ is the probability that all bidders other than $i$ have a value-signal less than $s$ or a budget less than $w$. This corresponds to the probability with which bidder $i$ wins the auction when she bids $\beta(s, w)$, as equilibrium bidding assumes. We can therefore regard Assumption A-4 as imposing a subtle limit on the rate of change in the probability of winning owing only to defeating opponents who have a smaller budget. If this probability increases too rapidly at some point $\hat{w}$ - for instance, due to an "atom"17 in the distribution of budgets - then as $b(s)$ crosses $\hat{w}, b^{\prime}(s)$ becomes undefined or negative and the continuous strategy we are considering can no longer be an equilibrium. At such bid levels, a bidder would have an incentive to drastically increase her bid to take advantage of others' budget constraints. ${ }^{18}$

In an interdependent-value setting, the preceding intuition continues to apply. However, it must be extended to incorporate the winner's curse. Defeating low-budget opponents is generally "good news" concerning the expected value of the item. Therefore in its fullest form, (11) additionally incorporates a weighted average controlling for these effects on an

\footnotetext{
${ }^{16}$ See Lemma B1 in the appendix.

${ }^{17} \mathrm{We}$ are assuming atom-less distributions of budgets, but the intuition in the extreme case of an atom in $G(w)$ is illuminating. Of course, there exist examples of a similar character when $G(w)$ admits a continuous density.

${ }^{18}$ Example 4 in the final section highlights this intuition explicitly.
} 
opponent-by-opponent basis.

Since the sufficient conditions in Assumption A-4 may be difficult to verify in practice, a simple (but exceptionally conservative) alternative is that

$$
g(w)(N-1) \mathbb{E}\left[u\left(1, Y_{1}, \ldots, Y_{N-1}\right) \mid S=1\right]<1
$$

We demonstrate the sufficiency of (12) in the online appendix. Effectively, it bounds $g(w)$ and places a uniform limit on the concentration of budget constraints in the relevant range of bids. Of course, this limit is not necessary for equilibrium existence as shown by Example 1 which does not meet this requirement.

Necessity A natural question to pose is to what extent our assumptions are necessary to support a continuous symmetric equilibrium? First, any assumptions concerning the differentiability of relevant functions are needed to ensure that the differential approach we adopt is possible. We consider such conditions to be economically innocuous. It is therefore more apt to examine the extent to which Assumption A-4 is necessary since it is the most unusual of the proposed conditions.

First suppose that $\xi(s, \underline{w} \mid s)<0$ in a neighborhood of $\tilde{s}_{\alpha}$. In this situation, the solution $b(s)$ cannot be extended continuously to bids in the range above $\underline{w}$. All solutions to the differential equation (7) will be decreasing in a neighborhood immediately above $\underline{w}$ and near $\tilde{s}_{\alpha}$. In this regard, Assumption A-4(2) cannot be relaxed while ensuring an equilibrium in continuous strategies.

From a formal point of view Assumption A-4(1) is not necessary for the existence of the equilibrium that we identify. From a practical perspective we view it as necessary. It is the weakest assumption that guarantees increasing solutions to $(7)$ on the domain $\left[\tilde{s}_{\alpha}, 1\right]$ without referring to the solution of (7) itself, which we view as too far removed from model primitives to be economically meaningful. At minimum, A-4(1) enjoys an economic interpretation, which we view as plausible. Weaker statements in lieu of Assumption A-4(1) would allow $\xi(s, \cdot \mid s)$ to fail its single-crossing condition provided the failure did not adversely affect the solution to (7) that is intended to be used in defining equilibrium bidding.

\subsection{Comparative Statics}

To place the equilibrium in context and to foster intuition for its properties we investigate several comparative statics. Throughout we focus on the effect of changes of the environment 
on changes in individual bidder behavior.

\section{Changes in the Distribution of Budgets}

It is natural to assume that the distribution of bidders' budgets may vary with broader economic and social conditions. More austere times may imply agents have on average less resources to expend on the contest; an economic boom may encourage profligacy. Surprisingly, however, exogenous "uniform" changes in the distribution of budgets may lead to a non-uniform adjustment in the players' bidding strategies. Some types of bidders may bid more, while others may bid less.

For a concrete example, consider a change in the environment that makes budget constraints more lax on average. In principle, this relaxation can lead to two competing effects. First, when budget constraints are relaxed, bidders may be encouraged to bid moreconstraints on competition have been softened and its natural to posit that bids will rise. The countervailing force, however, draws on the amelioration of the winner's curse associated with budget constraints. Conditional on winning, the item is of relatively higher value when budget constraints bind since there is a good chance of having defeated a budget-constrained opponent. Relaxing budget constraints dampens this effect. The result would tend to pull bids down. In the context of the second-price auction, Fang and Parreiras (2002) conclude that the latter effect can dominate. As a result, they are able to derive an unambiguous comparative static in the second-price auction.

In the all-pay auction, however, there does not exist a simple ordering of equilibrium strategies as we change $G$. This is true even under very restrictive stochastic orders. To appreciate this conclusion, suppose $N=2$ and fix a distribution of budgets $G$ on $[\underline{w}, \bar{w}]$ where $\bar{\alpha}<\bar{w}$. As shown by Lemma B4 in the appendix, the equilibrium bidding strategy in this case will be bounded above by $\bar{\alpha}$. Consider a family of distribution functions indexed by $a \geq 1$ defined as $G_{a}(w) \equiv G(w)^{a}$. If $a^{\prime}>a$, then $G_{a^{\prime}}$ likelihood-ratio dominates $G_{a} \cdot{ }^{19}$ Intuitively, higher values of $a$ imply more relaxed budget constraints as larger realizations of $W_{i}$ are more common. Denote by $\beta_{a}(s, w)=\min \left\{b_{a}(s), w\right\}$ an equilibrium strategy parameterized by $a$ and meeting the conditions identified in our analysis. Suppose for $a=1$, the auction admits an equilibrium $\beta_{1}$. Since $g(w)$ is bounded, for all $a$ sufficiently large $1-g(w) a G(\bar{\alpha})^{a-1}>0$ for all $w \in[\underline{w}, \bar{\alpha}]$. Therefore, for $a$ sufficiently large, $\beta_{a}$ will define an equilibrium when budgets are distributed according to $G_{a}$. By examining the main differential equation defining $b_{a}(s)$

\footnotetext{
${ }^{19}$ See Krishna (2002, p. 260).
} 
as $a \rightarrow \infty$, we see that

$$
\frac{\left(1-G(b)^{a}\right) v_{1}(s, s) f_{1}(s \mid s)}{1-a g(b) G(b)^{a-1} \int_{s}^{1} v_{1}(s, y) f_{1}(y \mid s) d y} \rightarrow v_{1}(s, s) f_{1}(s \mid s)
$$

uniformly for all $s$ and $b \leq \bar{\alpha}$. Therefore $b_{a}(s) \rightarrow \int_{0}^{s} v_{1}(y, y) f_{1}(y \mid y) d y$, as expected. Recall however that for each $a, b_{a}(s)>\alpha(s)$ for $s$ immediately to the right of $\tilde{s}_{\alpha}$ while (generically) $b_{a}(1)<\bar{\alpha}$. Therefore a bidder's strategy adjustment as budget constraints are relaxed is not monotone across types. In general $b_{a}(\cdot)$ is neither greater nor less than $b_{a^{\prime}}(\cdot)$ for $a^{\prime} \neq a$. Thus, the same qualitative ordering that exists for the second-price auction does not carry over to the case of the all-pay auction.

\section{Changes in the Bidder Population}

How will changes in the bidder population affect the auction's equilibrium? While original studies of auctions with budget constraints, such as Che and Gale (1998b), allowed for variation in the number of bidders, comparative statics exploring the sensitivity of equilibrium to changes in $N$ were not pursued systematically. The studies by Fang and Parreiras (2002) and Kotowski (2013) limited attention to the case of two bidders. Surprisingly, in our model the existence of an equilibrium in the canonical class is very sensitive to the number of bidders in the auction. This conclusion applies even in an independent, private-value setting.

Fix an auction environment with private values and suppose there is an equilibrium of the form $\beta(s, w)=\min \{b(s), w\}$ for some $N \geq 2$. Changing $N$ can lead to two main violations of Assumption A-4. First, due to a change in $N$ at the (new) critical value $\tilde{s}_{\alpha}$, the (new) expression (11) is such that $\xi\left(\tilde{s}_{\alpha}, \underline{w} \mid \tilde{s}_{\alpha}\right)<0$, which violates Assumption A-4(2). Second, even if A-4(2) is satisfied, following a change in the number of bidders $\xi(s, w \mid s)$ may instead violate the single-crossing condition from Assumption A-4(1). The violation can preclude the existence of a strictly increasing solution to (7) for all $s \geq \tilde{s}_{\alpha}$. We illustrate both failures with an example. The example assumes private values and so the documented ill-behavior of the equilibrium strategy is not a consequence of value-interdependence.

Example 2. Suppose there are $N$ bidders with private values, i.e. $u\left(s_{i}, \mathbf{s}_{-i}\right)=s_{i}$. Valuesignals are distributed uniformly and independently on the unit interval. Budgets are distributed independently according to the distribution $G(w)=1-\exp (-4(w-\underline{w}))$ with support $[\underline{w}, \infty)$. Choose $\underline{w}=0.1$.

Adding a subscript to emphasize the dependence on $N$, we can express $b_{N}(s)$ for bids 
below $\underline{w}$ as

$$
b_{N}(s)=\frac{N-1}{N} s^{N} .
$$

The associated critical value is $\tilde{s}_{\alpha, N}=\sqrt[N]{\frac{1}{10} \cdot \frac{N}{N-1}}$. Similarly, for each $N \geq 2$ we can calculate $\xi(s, w \mid s)$ to be

$$
\xi_{N}(s, w \mid s)=1+4(N-1)(s-1) s e^{\frac{2}{5}-4 w}\left((s-1) e^{\frac{2}{5}-4 w}+1\right)^{N-2}
$$

Again, we have used an $N$ subscript to emphasize this function's dependence on $N$. We consider three cases:

1. Suppose $N=2$, then $\xi_{2}(s, w \mid s)=4(s-1) s e^{\frac{2}{5}-4 w}+1$, which is strictly positive for all $(s, w) \in[0,1] \times[\underline{w}, \infty)$ except at the point $(s, w)=\left(\frac{1}{2}, \frac{1}{10}\right)$ where it is zero. Since $\tilde{s}_{\alpha, 2}=$ $\frac{1}{\sqrt{5}} \approx 0.447$, Assumption A-4 is satisfied and a symmetric equilibrium in canonical strategies exists.

2. Keeping the environment otherwise the same, suppose $N=3$. Now $\tilde{s}_{\alpha, 3}=\frac{\sqrt[3]{\frac{3}{5}}}{2^{2 / 3}} \approx 0.531$. At this value, $\xi_{3}\left(\tilde{s}_{\alpha, 3}, \underline{w} \mid \tilde{s}_{\alpha, 3}\right)=\frac{11}{5}-2\left(\frac{6}{5}\right)^{2 / 3}<0$. This is a violation of Assumption A-4(2) and a continuous extension of $b(s)$ at $\tilde{s}_{\alpha, 3}$ into the range above $\underline{w}$ is not possible. We note that A-4(1) is otherwise satisfied.

3. Finally, suppose $N=10$. In practical terms this would be a setting with a large number of bidders. Now, $\tilde{s}_{\alpha, 10}=\frac{1}{\sqrt[5]{3}} \approx 0.803$ and $\xi_{10}\left(\tilde{s}_{\alpha, 10}, \underline{w} \mid \tilde{s}_{\alpha, 10}\right)=5-4 \sqrt[5]{3} \approx$ $0.017>0$. Thus, Assumption A-4(2) is met. However, Assumption A-4(1) fails. We illustrate this failure with Figure 2. The figure shows the function $b(s)$ along with its solution satisfying the boundary condition $b\left(\tilde{s}_{\alpha, 10}\right)=\underline{w} \cdot{ }^{20}$ This extension of $b(s)$ above $\underline{w}$ necessarily needs to traverse a region, illustrated in gray, where $\xi_{10}(s, w \mid s)<0$. Therefore, there does not exist a strictly increasing solution to (10) as required.

The main implication stemming from Example 2 concerns the possibilities and opportunities for inference in auction environments where bidders may be budget constrained. While there does not exist a good theory of inference and identification in auctions with budget constraints (and it is far beyond the scope of this study to develop one), changes in $N$ are a common source of variation exploited in empirical auction studies. ${ }^{21}$ Fully exploiting this

\footnotetext{
${ }^{20}$ We construct Figure 2 by computing and plotting the inverse of $b(s)$. This technique allows us to accommodate instances where $b^{\prime}(s)=\infty$ for some $s$.

${ }^{21}$ See Athey and Haile (2007) for a recent survey of identification in auction models. See Bajari and Hortaçsu (2005) for an implementation.
} 


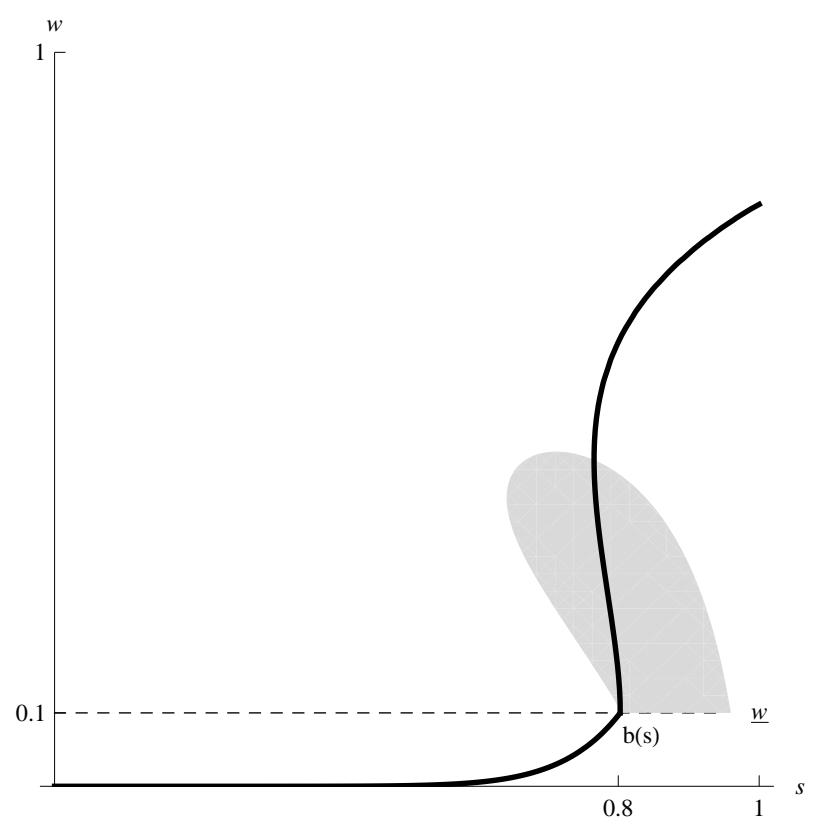

Figure 2: A failure of Assumption A-4. The gray region is the set $\left\{(s, w): \xi_{10}(s, w \mid s)<0\right\}$. Elsewhere, $\xi_{10}(s, w \mid s) \geq 0$.

variation in auctions with budget constraints may be problematic (or at best challenging) due to the qualitative differences of equilibrium bidding as the environment changes with $N$. For example, for some values of $N$ (depending on the distribution of budgets and valuations), one would not be able to employ first-order conditions to fully characterize a bidder's optimal bid. Much more research is required to develop precise conclusions and restrictions accounting for such concerns.

\section{Public Signals}

Suppose prior to bidding players observe the realization of some public signal $S_{0}$, which is affiliated with bidders' value-signals. We may further suppose that each bidder's payoff depends on the value of this signal, i.e. $V_{i}=u\left(S_{0}, S_{i}, S_{-i}\right)$. For example, this signal may be some information released non-strategically by the auctioneer or some widely available piece of economic news. Before examining how bidding may depend on the public signal, we distinguish two (non-exclusive) types of public signals that the bidders may observe.

Definition 1. The public signal $S_{0}$ is value-relevant if for a.e. $\left(s_{i}, \mathbf{s}_{-i}\right), u\left(s_{0}, s_{i}, \mathbf{s}_{-i}\right)$ is strictly increasing in $s_{0}$. $S_{0}$ is said to be value-irrelevant if for all $\left(s_{i}, \mathbf{s}_{-i}\right), u\left(s_{0}, s_{i}, \mathbf{s}_{-i}\right)$ is constant in $s_{0}$. 
Definition 2. Let $\bar{s}_{0}$ and $\underline{s}_{0}$ be two realizations of $S_{0}$. The public signal $S_{0}$ is informationrelevant if there exists a set $A, \operatorname{Pr}\left[S_{-i} \in A\right]>0$, such that $\bar{s}_{0} \neq\left.\underline{s}_{0} \Longrightarrow h\left(\cdot \mid s_{i}, \bar{s}_{0}\right)\right|_{A}<$ $\left.h\left(\cdot \mid s_{i}, \underline{s}_{0}\right)\right|_{A}$ or $\left.h\left(\cdot \mid s_{i}, \bar{s}_{0}\right)\right|_{A}>\left.h\left(\cdot \mid s_{i}, \underline{s}_{0}\right)\right|_{A}$.

A signal that is value-relevant conveys information about the value of the item directly; its realized value is effectively a parameter of a bidder's utility function. An informationrelevant signal is correlated with other bidders' private information. Therefore, it conveys additional information about others' signals beyond the information contained already in $S_{i}$. While nothing precludes a signal from being both value- and information-relevant-we believe that most signals embody both characteristics - we will focus only on extreme cases where public signals are either value- or information-relevant, but not both. This dichotomy allows us to emphasize the competing effects of information in the all-pay auction. Signals that are purely value-relevant encourage bidders to respond in the intuitive manner - "good news" will encourage uniformly more aggressive bidding. In contrast, high realizations of signals that are solely information-relevant may be a discouragement. Some types of bidders place lower bids as a result.

Theorem 2. Suppose the conditions of Theorem 1 are satisfied. Let $\bar{s}_{0}>\underline{s}_{0}$ be high and low realizations of a public signal $S_{0}$ observable to all bidders. Let $\bar{\beta}(s, w)$ be the symmetric equilibrium strategy in the all-pay auction when the public signal is high. Define $\underline{\beta}(s, w)$ analogously when the realized public signal is low.

1. If the public signal is value-relevant but $h\left(\cdot \mid \cdot, \bar{s}_{0}\right)=h\left(\cdot \mid \cdot \underline{s}_{0}\right)$, then $\bar{\beta}(s, w) \geq \underline{\beta}(s, w)$.

2. If the public signal is value-irrelevant but information relevant, then there exists an $\hat{s}>0$ such that for all $0<s<\hat{s}, \bar{\beta}(s, w) \leq \underline{\beta}(s, w)$.

The intuition behind Theorem 2 is simple. First, consider the case of purely value-relevant information. Noting the preceding discussion, and viewing $s_{0}$ as a parameter entering $u$ it is clear that our equilibrium characterization remains the same with statements conditional on $s_{0}$ replacing the unconditional statements. An implicit assumption, of course, is that changes in $s_{0}$ are sufficiently small to ensure that we maintain a symmetric equilibrium in canonical strategies. With this qualification in mind, the associated comparative static is intuitive.

In turning to information-relevant signals, we observe a different reaction. This conclusion is independent of the presence of budget constraints per se but is instead a general feature 
of the all-pay auction. ${ }^{22}$ The intuition is straightforward. Conditional on observing a high public signal $\bar{s}_{0}$ bidder $i$ can infer that her opponent likely has a high signal and will in consequence bid high. A high bid by the opponent decreases the probability with which bidder $i$ wins the auction, discouraging her from bidding aggressively. In contrast, if the public signal also has a direct effect on a bidder's value for the item, the resulting boost in expected payoff may be enough to counteract the discouragement effect.

\section{The War of Attrition}

Given that the first-price, second-price, and all-pay auctions have symmetric equilibria of the form $\beta(s, w)=\min \{b(s), w\}$, a natural conjecture is that the war of attrition also has an equilibrium in canonical strategies. In this section we extend our baseline model to accommodate this auction format. We maintain our assumptions concerning the environment from Section 1. Again, bidders will simultaneously submit bids and the highest bidder will be deemed the winner. (Ties are resolved with a uniform randomization.) Unlike the preceding analysis, in the (static) war of attrition the winning bidder makes a payment equal to the second-highest bid. All losing bidders continue to incur a cost equal to their bid. Sometimes, this auction format is called the second-price, all-pay auction. Our static treatment of the war of attrition mirrors the analysis in Krishna and Morgan (1997). Therefore, we do not model the war of attrition as an extensive game where bidders sequentially submit additional (incremental) bids. Leininger (1991) and Dekel et al. (2006) consider such models with budget limits and perfect information. Hörisch and Kirchkamp (2010) present an experimental comparison of static and dynamic implementations of the war of attrition.

Many of the qualitative features of the all-pay auction's equilibrium find natural analogues in the equilibrium of the war of attrition. The major distinction is that under a very mild technical condition the war of attrition features a uniform amplification of unconstrained bids following the introduction of budget constraints. Bidders with large budgets will increase their equilibrium bid relative to their equilibrium bid in the same environment absent budget constraints. In the all-pay auction, such an amplification was present only for a subset of types with intermediate value-signals.

As the derivation of the equilibrium strategy in the war of attrition parallels that from the all-pay auction, we abbreviate our discussion accordingly. Before pursuing the details,

\footnotetext{
${ }^{22}$ We have not found this comparative static noted before in the literature on symmetric all-pay auctions. Many studies, however, note similar "discouragement effects" in bidding games and contests, particularly when players are ex ante asymmetric. See Dechenaux et al. (2012) for a survey.
} 
however, recall that under suitable assumptions Krishna and Morgan (1997) show that the war of attrition without budget constraints has a symmetric equilibrium where all bidders adopt the strategy

$$
\omega(s)=\int_{0}^{s} \frac{v_{N-1}(y, y) f_{N-1}(y \mid y)}{1-F_{N-1}(y \mid y)} d y .
$$

This strategy has two important properties. First, it is strictly increasing. Second, it is not bounded: $\lim _{s \rightarrow 1^{-}} \omega(s)=\infty$ (Krishna and Morgan, 1997, Proposition 1). Hence, there exists a unique $\tilde{s}_{\omega}$ such that $\omega\left(\tilde{s}_{\omega}\right)=\underline{w}$. This parameter is the analogue of $\tilde{s}_{\alpha}$ from our study of the all-pay auction.

We will identify an equilibrium in the war of attrition with budget constraints which assumes the form $\beta(s, w)=\min \{b(s), w\}$. Again, $b(s)$ will be defined as an increasing solution to a differential equation. It will be piecewise differentiable. The derivation of $b(s)$ in the war of attrition is somewhat more complicated than our argument for the all-pay auction since a winning bidder's payment is uncertain at the time of bidding. To begin, suppose $b(s)$ is a strictly increasing function. Let

$$
F_{k}(x \mid s)=\underbrace{\int_{0}^{1} \cdots \int_{0}^{1}}_{N-1-k} \underbrace{\int_{0}^{x} \cdots \int_{0}^{x}}_{k} h\left(y_{1}, \ldots, y_{N-1} \mid s\right) d y_{1} \cdots d y_{N-1}
$$

and define

$$
\hat{H}(x, b(x) \mid s)=\sum_{k=0}^{N-1} \gamma_{k}(b(x)) F_{k}(x \mid s)
$$

If all bidders $j \neq i$ are following a bidding strategy $\beta(s, w)=\min \{b(s), w\}, \hat{H}(x, b(x) \mid s)$ is the probability that all bidders $j \neq i$ have a type $\left(s_{j}, w_{j}\right)$ such that $\beta\left(s_{j}, w_{j}\right)<b(x)$. We can write the expected utility of a bidder placing the bid $b(x)$, as

$$
\begin{aligned}
U_{i}(b(x) \mid s, w)= & \sum_{k=0}^{N-1} \gamma_{k}(b(x)) z_{k}(x \mid s)-(1-\hat{H}(x, b(x) \mid s)) b(x) \\
& \quad-\int_{0}^{\tilde{s}_{\omega}} \omega(y) f_{N-1}(y \mid s) d y-\left.\int_{\tilde{s}_{\omega}}^{x} b(y) \frac{d}{d z} \hat{H}(z, b(z) \mid s)\right|_{z=y} d y
\end{aligned}
$$

The first term is the expected benefit of winning the auction. The second term is the payment the bidder must make if she loses the auction. This equals her own bid. The third and fourth terms account for the payment she makes when she wins the auction.

Assuming appropriate differentiability, we can compute $\left.\frac{d}{d x} U_{i}(b(x) \mid s, w)\right|_{x=s}=0$ leading 
to the differential equation

$$
b^{\prime}(s)=\frac{\sum_{k=0}^{N-1} \gamma_{k}(b(s)) z_{k}^{\prime}(s \mid s)}{1-\hat{H}(s, b(s) \mid s)-\sum_{k=0}^{N-1} \gamma_{k}^{\prime}(b(s)) z_{k}(s \mid s)},
$$

which characterizes $b(s)$.

Mirroring our analysis of the all-pay auction, we again propose two assumptions - alternatives to Assumptions A-3 and A-4 - that are sufficient to ensure that our preceding arguments are reflective of a symmetric equilibrium. The first assumption places a limit on the relative degree of affiliation and generalizes a condition proposed by Krishna and Morgan (1997). The second assumption is the analogue of Assumption A-4. It structures the joint distribution of value-signals and budgets.

Assumption A-5. Let

$$
\Phi(x, w \mid s)=\sum_{k=1}^{N-1} \frac{\gamma_{k}(w)\left(1-F_{k}(x \mid s)\right)}{\sum_{k^{\prime}=1}^{N-1} \gamma_{k^{\prime}}(w)\left(1-F_{k^{\prime}}(x \mid s)\right)}\left(\frac{v_{k}(s, x) f_{k}(x \mid s)}{1-F_{k}(x \mid s)}\right) .
$$

$\Phi(x, w \mid \cdot):[0,1] \rightarrow \mathbb{R}$ is non-decreasing

Like Assumption A-3, Assumption A-5 is a restriction on the relative degree of affiliation among value-signals. It always holds if value-signals are independent. If $w \leq \underline{w}$, then (15) reduces to

$$
\frac{v(\cdot, x) f_{N-1}(x \mid \cdot)}{1-F_{N-1}(x \mid \cdot)}:[0,1] \rightarrow \mathbb{R}
$$

being non-decreasing for each $x$. This is Krishna and Morgan's condition supporting (13) as an equilibrium strategy when budget constraints are not present. Hence Assumption A-5 is a generalization of their original assumption. (15) simplifies similarly when $N=2$. To emphasize the parallel with Assumption A-3 above, we stated A-5 as a weighted average; hence, the expression is not fully simplified. This notation stresses the interaction between the number of bidders and the limit on affiliation that needs to hold given different subsets of bidders.

Assumption A-6. Let

$$
\Xi(x, w \mid s)=1-\frac{\sum_{k=0}^{N-1} \gamma_{k}^{\prime}(w) z_{k}(x \mid s)}{\sum_{k=1}^{N-1} \gamma_{k}(w)\left(1-F_{k}(x \mid s)\right)} .
$$

$\Xi(x, w \mid s)$ satisfies the following properties: 
1. For every $s \geq \tilde{s}_{\omega}$, there exists $w_{s}, \underline{w} \leq w_{s}<\bar{w}$ such that $w<w_{s} \Longrightarrow \Xi(s, w \mid s)<0$ and $w \in\left(w_{s}, \bar{w}\right) \Longrightarrow \Xi(s, w \mid s)>0$.

2. There exists $\epsilon>0$ such that $s \in\left(\tilde{s}_{\omega}-\epsilon, \tilde{s}_{\omega}+\epsilon\right) \Longrightarrow \Xi(s, \underline{w} \mid s)>0$.

3. When $x \geq \tilde{s}_{\omega}, \Xi(x, w \mid \cdot):[0,1] \rightarrow \mathbb{R}$ is non-increasing.

The conditions in Assumption A-6 are direct adaptations of the conditions introduced in Assumption A-4. Their interpretation and roles are also analogous. Some simplifications of Assumption A-6 are possible in special cases. For example, Assumption A-6(3) is satisfied automatically if value-signals are independent.

Theorem 3 collects the preceding assumptions and offers sufficient conditions for a symmetric equilibrium in the war of attrition in canonical strategies. The equilibrium strategy resembles the equilibrium of the all-pay auction. Low value-signal bidders follow the usual no-budget-constraints equilibrium strategy, $\omega(s)$. Only for bids above $\underline{w}$ will the change in incentives introduced by budget constraints modify equilibrium behavior. Unlike the all-pay auction, bidders with sufficiently large value-signals will desire to expend an arbitrarily large amount in equilibrium. If $\bar{w}=\infty$, then the equilibrium strategy is unbounded.

Theorem 3. Suppose Assumptions A-1, A-2, A-5, and A-6 hold. Then there exists a symmetric equilibrium in continuous strategies in the war of attrition. In this equilibrium, all bidders follow the strategy $\beta(s, w)=\min \{b(s), w\}$ defined as follows:

- For all $s<\tilde{s}_{\omega}, b(s)=\omega(s)$ where $\omega(s)$ is defined in $(13)$.

- For all $\tilde{s}_{\omega} \leq s<\hat{s}_{\omega}, b(s)$ is the solution to the differential equation (14) satisfying the boundary condition $b\left(\tilde{s}_{\omega}\right)=\underline{w}$.

- For all $s \geq \hat{s}_{\omega}, b(s)=\bar{w}$.

The value $\tilde{s}_{\omega}$ is the unique solution to $\underline{w}=\omega\left(\tilde{s}_{\omega}\right)$ while $\hat{s}_{\omega}$ is the smallest value such that $\lim _{s \rightarrow \hat{s}_{\omega}^{-}} b(s)=\bar{w}$.

The following example illustrates an equilibrium in the war of attrition.

Example 3. Suppose $N=2$ and $S_{i} \stackrel{\text { i.i.d. }}{\sim} U[0,1]$. Let $u\left(s_{i}, s_{j}\right)=\left(s_{i}+s_{j}\right) / 2$. Suppose $G(w)=1-e^{-(w-\underline{w})}$ where $\underline{w}=-\frac{7}{20}+\log \left(\frac{20}{13}\right) \approx 0.081$. With these parameters, our equilibrium strategy in the war of attrition is $\beta(s, w)=\min \{b(s), w\}$ where

$$
b(s)=\left\{\begin{array}{ll}
-s-\log (1-s) & \text { if } s \leq \frac{7}{20} \\
\int_{\frac{7}{20}}^{s} \frac{4 y}{3(y-1)^{2}} d y+\underline{w} & \text { if } s>\frac{7}{20}
\end{array} .\right.
$$




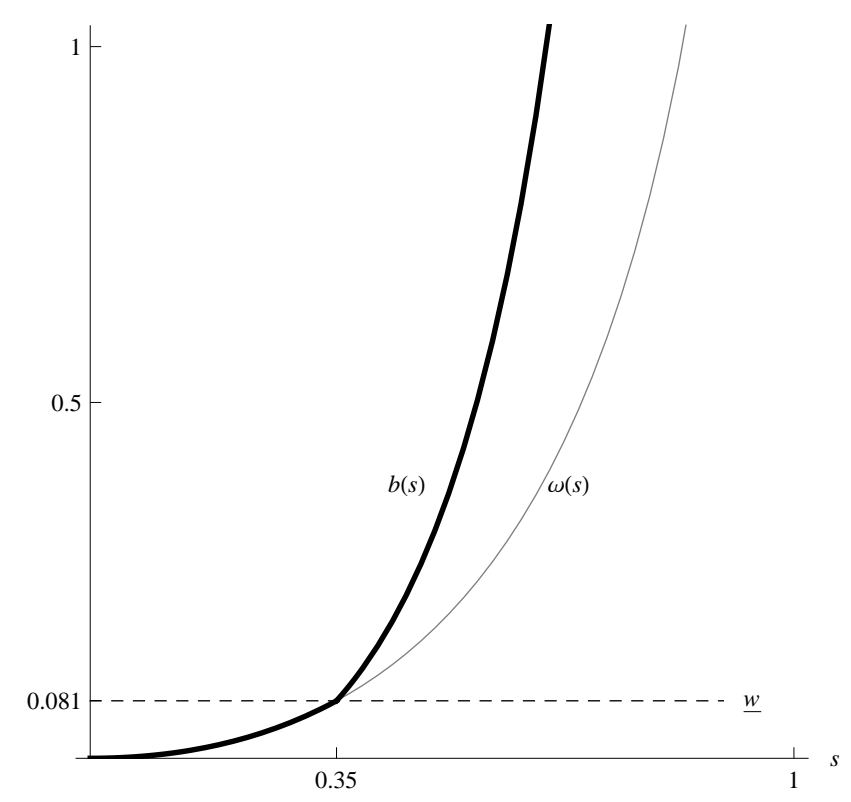

Figure 3: The functions $b(s)$ and $\omega(s)$ in the characterization of equilibrium bidding in Example 3. Both functions are not bounded.

For $s>7 / 20$, we can integrate the above expression to arrive at

$$
b(s)=\frac{1040(s-1) \log (1-s)+1820(s-1) \log \left(\frac{20}{13}\right)-1873 s+833}{780(s-1)}
$$

For comparison, Figure 3 presents the functions $b(s)$ and $\omega(s)=-s-\log (1-s)$.

As seen in Example 3, bidders with a value-signal of only 0.65 desire to commit to a bid greater than 1, which is the maximum possible value of the available prize. Such "overbidding" is a particular feature of the war of attrition, with or without budget constraints (Albano, 2001). The example suggests that the introduction of budget constraints amplifies the overbidding phenomenon further. This observation is formalized by the following corollary.

Corollary 2. Under the conditions of Theorem 3:

1. $\lim _{s \rightarrow \tilde{s}_{\omega}^{+}} b^{\prime}(s)>\lim _{s \rightarrow \tilde{s}_{\omega}^{-}} b^{\prime}(s)$.

2. If $\frac{f_{k}(s \mid s)}{1-F_{k}(s \mid s)} \geq \frac{f_{N-1}(s \mid s)}{1-F_{N-1}(s \mid s)}$ for all $k,{ }^{23}$ then for all $s$ such that $b(s)<\bar{w}, b(s) \geq \omega(s)$.

${ }^{23}$ This condition is satisfied when $S_{i} \stackrel{i . i . d .}{\sim} U[0,1]$. 
The equilibrium in the war of attrition exhibits similar comparative statics to the all-pay auction. Again, the equilibrium strategy identified here will converge to the equilibrium in an environment without budget constraints if the constraints are relaxed. Additionally, the same bidder-level comparative statics apply concerning information revelation. The distinction between value-relevant and information-relevant public signals continues to be important.

Theorem 4. Suppose the conditions of Theorem 3 are satisfied. Then the conclusions of Theorem 2 apply to the war of attrition.

\subsection{Comparing the All-Pay Auction and the War of Attrition}

We conclude our discussion of the war of attrition with a brief comparison to the all-pay auction. Naturally, we restrict attention to environments where the all-pay auction has an equilibrium of the form $\beta_{\alpha}(s, w)=\min \left\{b_{\alpha}(s), w\right\}$ and the war of attrition has an equilibrium of the form $\beta_{\omega}(s, w)=\min \left\{b_{\omega}(s), w\right\} .{ }^{24}$ Both $\beta_{\alpha}$ and $\beta_{\omega}$ are assumed to exhibit the characteristics identified in our preceding analysis. Our first comparison considers an ordering of the bidding strategies.

Theorem 5. $\beta_{\omega}(s, w) \geq \beta_{\alpha}(s, w)$ for all $(s, w)$.

Noting Theorem 5, we can employ the arguments in Che and Gale (1998b) and also outlined in Krishna (2002) to conclude that the all-pay auction will be more efficient on average than the war of attrition when ex-post preferences reflect the ordering of bidder's value-signals.

With regards to revenues, there does not exist a general revenue ranking between the war of attrition and the all-pay auction in the presence of budget constraints and affiliated valuations. One can draw this conclusion by documenting the results in extreme cases. First, suppose that budget constraints are very lax. For example, suppose budgets are distributed according to the exponential distribution with a mean that is very large. Since budget constraints in this case are almost irrelevant, the equilibrium bids submitted in both formats are essentially the same as those submitted in the case of no budget constraints. Drawing on Krishna and Morgan (1997) we can conclude that in the presence of value interdependence, the war of attrition will revenue-dominate the all-pay auction.

\footnotetext{
${ }^{24}$ We employ $\alpha$ and $\omega$ subscripts to differentiate between the all-pay auction $(\alpha)$ and the war of attrition $(\omega)$.
} 
When budget constraints are more meaningful, and they constrain bidders with nonvanishing probability, the all-pay auction can generate more revenue. Consider the following case. Suppose there are two bidders and value signals are distributed independently according to the uniform distribution. Suppose budget constraints follow the exponential distribution $G(w)=1-e^{-(w-\underline{w})}$ on $[\underline{w}, \infty)$. Choose $\underline{w}=\log \left(\frac{10}{3}\right)-\frac{7}{10} \approx 0.5039$. Finally, assume bidders have private values: $u_{i}\left(s_{i}, s_{j}\right)=s_{i}$.

In this situation, budget constraints are (just) irrelevant in the case of the all-pay auction. The equilibrium strategy is $\beta_{\alpha}(s, w)=\min \left\{b_{\alpha}(s), w\right\}$ where $b_{\alpha}(s)=\frac{s^{2}}{2}$. The expected revenue in the all-pay auction is $R_{\alpha}=\frac{1}{3}$.

Since the bidding strategy in the war of attrition is not bounded, the introduced budget constraints will directly affect the equilibrium strategy. It is straightforward to show that the equilibrium bidding strategy is $\beta_{\omega}(s, w)=\min \left\{b_{\omega}(s), w\right\}$ where

$$
b_{\omega}(s)= \begin{cases}-s-\log (1-s) & \text { if } s<\frac{7}{10} \\ \int_{\frac{7}{10}}^{s} \frac{y}{(y-1)^{2}} d y+\log \left(\frac{10}{3}\right)-\frac{7}{10} & \text { if } s \geq \frac{7}{10}\end{cases}
$$

When $s>\frac{7}{10}$, we can write $b_{\omega}(s)$ in closed form as

$$
b_{\omega}(s)=\frac{s\left(\log \left(\frac{1000}{27}\right)-10\right)+7+\log (27)-3 \log (10)}{3(s-1)}+\log \left(\frac{10}{3}-\frac{10}{3} s\right)-\frac{7}{10} .
$$

A direct calculation for the revenue (see the online appendix) gives

$$
R_{\omega}=\frac{1}{1500}\left(527-270 e^{\frac{20}{3}} \int_{\frac{20}{3}}^{\infty} \frac{e^{-x}}{x} d x\right)
$$

The terms in $R_{\omega}$ are straightforward to approximate accurately to conclude that $R_{\omega}<0.328$. In this example the impact on revenue following the introduction of budget constraints is slight for two reasons. First, only a small fraction of bidders in the war of attrition are somehow directly impacted by the budget constraint. Additionally, some bidders adjust their bids upward (Corollary 2) which partially ameliorates the revenue decline. However, the adjustment is not sufficient to preclude a strict drop in revenue. Hence, $R_{\alpha}>R_{\omega}$.

While tractability has guided our discussion of revenues towards comparisons of extreme scenarios, its conclusions apply more generally. It is clear that we can modify our final example by perturbing the distribution of budgets slightly such that it has full support on $[0, \infty)$ without changing the conclusion. Similarly, one can perturb the distribution of 
value-signals such that they are strictly but "slightly" affiliated. For example, consider the distribution $h\left(s_{1}, s_{2}\right)=\frac{4}{1+4 k}\left(k+s_{i} s_{j}\right)$ on $[0,1]^{2}$ and let $k$ be very large. This change will not compromise the strict difference in expected revenue. Finally, one can introduce strict value interdependence by endowing bidders with the preferences $u\left(s_{i}, s_{j}\right)=(1-\epsilon) s_{i}+\epsilon s_{j}$. As we have shown, the boundary of the revenue dominance of one auction format over the other will lie somewhere in between the two extreme cases considered.

Our conclusions regarding revenues have implications concerning the efficacy of different fundraising procedures. Recently, Goeree et al. (2005) established the superiority of (lowestprice) all-pay auctions over, for example, standard winner-pay auctions and lotteries in raising funds for public goods or charity. ${ }^{25}$ The distinguishing feature of this application is that auction participants benefit from acquiring the good for sale and from the contributions raised for the charity. Goeree et al. argue that all-pay mechanisms are particularly good at harnessing the latter externality; hence, they generate greater revenues. To be specific they show that an appropriately calibrated lowest-price, all-pay auction raises the most revenue. In such a mechanism, the highest bidder wins the item but all bidders pay a price equal to the lowest submitted bid.

Our analysis qualifies Goeree et al.'s conclusion, at least when public-good externalities are small. ${ }^{26}$ When there are only two participants, the lowest-price, all-pay auction is the (static) war of attrition. As showed by our example, with private budget constraints the (first-price) all-pay auction is revenue superior. More generally, we anticipate that the presence of private budget constraints will render the lowest-price all-pay auction comparatively less attractive. Private budget constraints nearly always compromise the auction's efficiency and this inefficiency is exacerbated when equilibrium bids are on average higher. The net result is a decline in collected revenue. ${ }^{27}$

\section{Discontinuous Equilibria and Concluding Remarks}

Our study has focused on equilibria in continuous strategies; however, we have already seen that in many situations an equilibrium in continuous canonical strategies may not exist. This occurs primarily when the distribution of budgets is "too dense" at some point. When

\footnotetext{
${ }^{25}$ See also Morgan (2000). Corazzini et al. (2010) provide a recent experimental comparison of lotteries and all-pay auctions in a fundraising context.

${ }^{26}$ This would correspond to $\alpha \approx 0$ in Goeree et al. (2005).

${ }^{27} \mathrm{We}$ conjecture that in the presence of private budget constraints, the optimal auction in the setting of Goeree et al. (2005) will most often involve a $k$-th price all-pay auction where $1<k<N$.
} 
this is the case, equilibrium bidding will be more complicated. Discontinuous equilibrium strategies cannot be ruled out. While a full characterization of such equilibria is beyond the focus of this paper, we offer the following example that is suggestive of the qualitative features that can emerge. Except for the unusual budget distribution, the set-up of Example 4 parallels the environment of Example 1 discussed above. ${ }^{28}$

Example 4. Suppose there are two bidders each of whom observes a value-signal $S_{i} \stackrel{\text { i.i.d. }}{\sim}$. $U[0,1]$. Suppose that the item's ex post value to bidder $i$ is $u\left(s_{i}, s_{j}\right)=\left(s_{i}+s_{j}\right) / 2$. Suppose that budgets are distributed independently and identically in the following manner. With probability $1 / 2$ a bidder has a budget of $w_{i}=1 / 5$. With probability $1 / 2$, a bidder has a budget of $w_{i}=1$. For reference, we note that in the absence of budget constraints, the equilibrium bidding strategy in the all-pay auction is $\alpha(s)=s^{2} / 2$.

With private budget constraints equilibrium bidding in the all-pay auction will exhibit a discontinuity. In fact, the equilibrium strategy becomes

$$
\beta(s, w)= \begin{cases}\frac{s^{2}}{2} & s \leq \hat{s} \\ \frac{s^{2}}{4}+\frac{(2 \sqrt{10}-5)^{2}}{100} & \hat{s}<s<\hat{s}^{\prime}, w=\frac{1}{5} \\ \frac{1}{5} & \hat{s}^{\prime} \leq s, w=\frac{1}{5} \\ \frac{s^{2}}{4}+\frac{4 \sqrt{10}-9}{20} & \hat{s}<s, w=1\end{cases}
$$

where $\hat{s}=-1+2 \sqrt{\frac{2}{5}} \approx 0.265$ and $\hat{s}^{\prime}=-1+\sqrt{-\frac{36}{5}+16 \sqrt{\frac{2}{5}}} \approx 0.709$. A proof that this strategy characterizes a symmetric equilibrium is presented in the online appendix. The argument is somewhat tedious due to its case-by-case nature; technically, it is straightforward.

The key feature of this equilibrium is the discontinuity in the bidding strategy at $\hat{s}$. For relatively low value-signals - those below $\hat{s}$ - each bidder bids according to the no-budget constraints equilibrium strategy, much like they did in our analysis of continuous equilibria. However, at $\hat{s}$, a high-budget bidder has an incentive to increase her bid by a substantial amount. At this value-signal, a bidder with a large budget increases her bid discontinuously from $\frac{1}{2}(2 \sqrt{2 / 5}-1)^{2} \approx 0.035$ to 0.2 . The increased bid drastically increases her probability of winning since she can outbid all budget-constrained opponents. The associated intuition echoes our discussion concerning the non-existence of continuous equilibria when the distribution of budgets is sufficiently concentrated on a single point, as noted in section 2.1. The

\footnotetext{
${ }^{28}$ Kotowski (2013) constructs a similar example in application to the first-price auction. An important difference is that his example assumes private values while ours has interdependent values.
} 
discrete distribution of budgets is a particularly extreme example of this phenomenon.

While the discrete budget distribution in Example 4 places it outside of our baseline environment, it is nevertheless suggestive of the strategic tradeoffs present in auctions with budget constraints. The discrete budgets support the presence of discontinuous equilibrium bidding strategies; however, we emphasize that the discontinuous strategies are not necessarily artifacts of the budget discreteness. For example, Kotowski (2013) shows that under appropriate conditions the first-price auction with continuously distributed budgets has an equilibrium in discontinuous strategies exhibiting the qualitative features of the discretebudget model. We anticipate that a similar construction carries over to our model of the all-pay auction and the war of attrition.

We motivated our investigation by noting the range of applications that all-pay auctions have seen in the economics literature. They form the core of many models of lobbying, student competition, or innovation. In another interesting application Baye et al. (2005) use a cleverly generalized model of an all-pay auction to study the performance of different legal systems. Specifically they investigate whether "who pays" a case's legal fees matters for the level of expenditures on litigation. For instance, in the United States each party in a legal dispute typically pays its own legal fees. In contrast, in Britain the losing party is customarily required to cover the legal fees of the winner in addition to its own.

Although absent from their formal model, Baye et al. (2005) recognize that budget and liquidity constraints often shape the legal strategy of parties in a dispute. While our model lacks their flexible parameterization spanning various legal systems, our analysis does allow us to draw some preliminary conclusions. If we view the legal contest as a (first-price) all-pay auction $^{29}$ then the introduction of private budgets has mixed implications. Notably, some participants will spend more than they otherwise would. Others might spend less. The war of attrition is, of course, another credible model of a legal contest. Here our model offers a rather sobering conclusion. The mechanical effects of budget constraints notwithstanding, ${ }^{30}$ the possibility that opponents may be budget constrained may encourage even greater (planned) expenditures. The ability to take advantage of others' possible budget constraints is a strategic option that many litigants undoubtably exploit in practice. More importantly, however, the resulting inefficiency in the contest's outcome may emerge as a miscarriage of justice. While evaluating such implications is decidedly beyond our model's immediate

\footnotetext{
${ }^{29}$ This is how Baye et al. (2005) characterize the "American system." In their model, it corresponds to the parameterization $\alpha=\beta=1$.

${ }^{30}$ In the presence of budget constraints, some agents will hit their spending cap. This mechanically constrains their expenditure.
} 
scope, the inefficiency induced by practical constraints on bids is a salient, albeit underemphasized, fact. Further research - theoretical, empirical, and experimental - is required to better evaluate the relative performance of different auction mechanisms in constrained settings. 


\section{A Appendix: Expected Payoffs in the All-Pay Auction}

Suppose all bidders other than $i$ are following the strategy $\beta(s, w)=\min \{b(s), w\}$ where $b(s)$ is strictly increasing. A bid of $b(x)$ will defeat two classes of opponents. $k$ opponents will have a budget greater than $b(x)$ and $N-1-k$ opponents will have a budget less than $b(x)$. The probability of this event is $\gamma_{k}(b(x))=\left(\begin{array}{c}N-1 \\ k\end{array}\right) G(b(x))^{N-1-k}(1-G(b(x)))^{k}$. The valuesignal of the opponents who have a budget less than $b(x)$ can be arbitrary. The value-signal of the opponents who have a budget greater than $b(x)$ must have a value-signal less than $x$. Thus, the contribution to expected utility from this event is

$$
\gamma_{k}(b(x)) \underbrace{\int_{0}^{1} \cdots \int_{0}^{1}}_{N-1-k} \underbrace{\int_{0}^{x} \cdots \int_{0}^{x}}_{k} u\left(s, y_{1}, \ldots, y_{N-1}\right) h\left(y_{1}, \ldots, y_{N-1} \mid s\right) d y_{1} \cdots d y_{N-1}
$$

Owing to symmetry, we have assumed without loss of generality that opponents $1, \ldots, k$ are in the first group while the remain opponents are in the latter. We can simplify the preceding expression as follows:

$$
\begin{aligned}
& \gamma_{k}(b(x)) \underbrace{\int_{0}^{1} \cdots \int_{0}^{1}}_{N-1-k} \underbrace{\int_{0}^{x} \cdots \int_{0}^{x}}_{k} u\left(s, y_{1}, \ldots, y_{N-1}\right) h\left(y_{1}, \ldots, y_{N-1} \mid s\right) d y_{1} \cdots d y_{N-1} \\
& =\gamma_{k}(b(x)) \operatorname{Pr}\left[\bar{Y}_{k} \leq x \mid S=s\right] \mathbb{E}\left[u\left(S, Y_{1}, \ldots, Y_{N-1}\right) \mid S=s, \bar{Y}_{k} \leq x\right] \\
& =\gamma_{k}(b(x)) \int_{0}^{x} \mathbb{E}\left[u\left(S, Y_{1}, \ldots, Y_{N-1}\right) \mid S=s, \bar{Y}_{k}=y\right] f_{k}(y \mid s) d y \\
& =\gamma_{k}(b(x)) \int_{0}^{x} v_{k}(s, y) f_{k}(y \mid s) d y \\
& =\gamma_{k}(b(x)) z_{k}(x \mid s)
\end{aligned}
$$

Recalling that $z_{0}(x \mid s)=v_{0}(s, y)=\mathbb{E}\left[u\left(s, Y_{1}, \ldots, Y_{N-1}\right) \mid S=s\right]$, we can sum over $k$ to arrive at $U_{i}(b(x) \mid s, w)=\sum_{k=0}^{N-1} \gamma_{k}(b(x)) z_{k}(x \mid s)-b(x)$. Note that if $b(x)<\underline{w}$, then for all $k=0, \ldots, N-2, \gamma_{k}(b(x))=0$ and $\gamma_{N-1}(b(x))=1$. Thus, the above expression reduces to

$$
U_{i}(b(x) \mid s, w)=z_{N-1}(x \mid s)=\int_{0}^{x} v_{N-1}(s, y) f_{N-1}(y \mid s) d y-b(x) .
$$




\section{B Appendix: Proofs}

Throughout our formal arguments we use the properties of affiliated random variables freely. We refer the reader to Milgrom and Weber (1982) or Krishna (2002, Appendix D) for a detailed introduction to their properties.

Lemmas B1 and B2 present preliminary results that are used throughout our analysis.

Lemma B1. $\sum_{k=0}^{N-1} \gamma_{k}(w)^{\prime} z_{k}(x \mid s) \equiv g(w)(N-1) \sum_{k=0}^{N-2}\left(\begin{array}{c}N-2 \\ k\end{array}\right) G(w)^{N-2-k}(1-G(w))^{k}\left(z_{k}(x \mid s)-\right.$ $\left.z_{k+1}(x \mid s)\right)$.

Proof. To simplify notation, we let $G \equiv G(w), g \equiv g(w), z_{k} \equiv z_{k}(x \mid s)$, and $\gamma_{k} \equiv \gamma_{k}(w)$. Differentiating $\gamma_{k}(w)$ with respect to $w$ gives

$$
\gamma_{k}^{\prime}=\left(\begin{array}{c}
N-1 \\
k
\end{array}\right)(N-1-k) G^{N-2-k} g(1-G)^{k}-\left(\begin{array}{c}
N-1 \\
k
\end{array}\right) G^{N-1-k} g k(1-G)^{k-1} .
$$

Therefore,

$$
\begin{aligned}
\sum_{k=0}^{N-1} \gamma_{k}^{\prime} z_{k}= & \sum_{k=0}^{N-2}\left(\begin{array}{c}
N-1 \\
k
\end{array}\right)(N-1-k) G^{N-2-k} g(1-G)^{k} z_{k} \\
& -\sum_{k=1}^{N-1}\left(\begin{array}{c}
N-1 \\
k
\end{array}\right) k G^{N-1-k} g(1-G)^{k-1} z_{k} .
\end{aligned}
$$

For $k \leq N-2,\left(\begin{array}{c}N-1 \\ k\end{array}\right)(N-1-k)=\frac{(N-1) !}{(N-1-k) ! k !}(N-1-k)=(N-1) \frac{(N-2) !}{(N-2-k) ! k !}=(N-1)\left(\begin{array}{c}N-2 \\ k\end{array}\right)$ and for $k \geq 1,\left(\begin{array}{c}N-1 \\ k\end{array}\right) k=\frac{(N-1) !}{(N-1-k) !(k-1) !}=(N-1) \frac{(N-2) !}{(N-2-(k-1)) !(k-1) !}=(N-1)\left(\begin{array}{c}N-2 \\ k-1\end{array}\right)$. Shifting the index of summation,

$$
(N-1) g \sum_{k=1}^{N-1}\left(\begin{array}{c}
N-2 \\
k-1
\end{array}\right) G^{N-1-k}(1-G)^{k-1} z_{k}=(N-1) g \sum_{k=0}^{N-2}\left(\begin{array}{c}
N-2 \\
k
\end{array}\right) G^{N-2-k}(1-G)^{k} z_{k+1} .
$$


Hence,

$$
\begin{aligned}
\sum_{k=0}^{N-1} \gamma_{k}^{\prime} z_{k}= & g(N-1) \sum_{k=0}^{N-2}\left(\begin{array}{c}
N-2 \\
k
\end{array}\right) G^{N-2-k} g(1-G)^{k} z_{k} \\
& -g(N-1) \sum_{k=0}^{N-2}\left(\begin{array}{c}
N-2 \\
k
\end{array}\right) G^{N-2-k}(1-G)^{k} z_{k+1} \\
= & g(N-1) \sum_{k=1}^{N-1}\left(\begin{array}{c}
N-2 \\
k-1
\end{array}\right) G^{N-1-k}(1-G)^{k-1}\left(z_{k-1}-z_{k}\right) \\
= & g(N-1) \sum_{k=0}^{N-2}\left(\begin{array}{c}
N-2 \\
k
\end{array}\right) G^{N-2-k}(1-G)^{k}\left(z_{k}-z_{k+1}\right) .
\end{aligned}
$$

Lemma B2. Let $\mathbf{y}=\left(y_{1}, \ldots, y_{N-1}\right)$. Then,

1. For all $k \geq 1$,

$$
z_{k}(x \mid s)=\int_{0}^{x} v_{k}(s, y) f_{k}(y \mid s) d y=\underbrace{\int_{0}^{1} \cdots \int_{0}^{1}}_{N-1-k} \underbrace{\int_{0}^{x} \cdots \int_{0}^{x}}_{k} u(s, \mathbf{y}) h(\mathbf{y} \mid s) d y_{1} \cdots d y_{N-1} .
$$

2. For all $k, z_{k}(x \mid s)-z_{k+1}(x \mid s) \geq 0$ and therefore, $\sum_{k=0}^{N-1} \gamma_{k}^{\prime}(w) z_{k}(x \mid s) \geq 0$.

Proof. 1. Working from the definition of $z_{k}(x \mid s)$ :

$$
\begin{aligned}
z_{k}(x \mid s) & =\int_{0}^{x} v_{k}(s, y) f_{k}(y \mid s) d y \\
& =\int_{0}^{x} \mathbb{E}\left[u\left(S, Y_{1}, \ldots Y_{N}\right) \mid S=s, \bar{Y}_{k}=y\right] f_{k}(y \mid s) d y \\
& =\operatorname{Pr}\left[\bar{Y}_{k} \leq x \mid S=s\right] \mathbb{E}\left[u\left(S, Y_{1}, \ldots Y_{N}\right) \mid S=s, \bar{Y}_{k} \leq x\right] \\
& =\underbrace{\int_{0}^{1} \cdots \int_{0}^{1}}_{N-1-k} \underbrace{\int_{0}^{x} \cdots \int_{0}^{x}}_{k} u(s, \mathbf{y}) h(\mathbf{y} \mid s) d y_{1} \cdots d y_{N-1} .
\end{aligned}
$$

2. Noting symmetry, we can write

$$
z_{k}(x \mid s)-z_{k+1}(x \mid s)=\int_{x}^{1} \underbrace{\int_{0}^{1} \cdots \int_{0}^{1}}_{N-1-(k+1)} \underbrace{\int_{0}^{x} \cdots \int_{0}^{x}}_{k} u(s, \mathbf{y}) h(\mathbf{y} \mid s) d y_{1} \cdots d y_{N-2} d y_{N-1} .
$$


Whenever $0<x<1$, the preceding expression is strictly positive.

Lemma B3 is used in the proof of Theorem 1. Remark B1 addresses a closely related point concerning valid solutions to the differential equation that characterizes equilibrium bidding.

Lemma B3. The differential equation

$$
b^{\prime}(s)=\frac{\sum_{k=0}^{N-1} \gamma_{k}(b(s)) z_{k}^{\prime}(s \mid s)}{1-\sum_{k=0}^{N-1} \gamma_{k}^{\prime}(b(s)) z_{k}(s \mid s)}
$$

has a strictly increasing solution $b(s):\left[\tilde{s}_{\alpha}, 1\right] \rightarrow[\underline{w}, \bar{w}]$ satisfying the boundary condition $b\left(\tilde{s}_{\alpha}\right)=\underline{w}$.

Proof. Since all of the terms in (B1) are continuous, this lemma follows from standard results in the theory of ordinary differential equations. It is readily verified that $b^{\prime}(s)$ is strictly positive for all $s \in\left[\tilde{s}_{\alpha}, 1\right]$ due to Assumption A-4. The basic intuition is presented in Figure B1. $\{(s, b): \xi(s, b \mid s)=0\}$, denoted by dashed curves, is a set of points where $b^{\prime}(s)$ is not defined and solutions to (B1) approach vertically. In grey regions, all solutions to (B1) are downward sloping while in the white region $b^{\prime}(s)>0$.

We need to however verify that the solution meeting the boundary condition $b\left(\tilde{s}_{\alpha}\right)=\underline{w}$ is defined for all $s \in\left[\tilde{s}_{\alpha}, 1\right]$. The sole alternative is that for some $\tilde{s}_{\alpha}^{\prime}<1, \lim _{s \rightarrow \tilde{s}_{\alpha}^{\prime}} b(s)=\bar{w}$. This possibility is ruled out, however, by noting that the function $\tilde{b}(s)=\bar{w}$ is a solution to (B1) satisfying the boundary condition $\tilde{b}\left(\tilde{s}_{\alpha}\right)=\bar{w}$. Thus, $b(s)$ is bounded above by $\bar{w}$ and the solution $b(s)$ has a maximal domain of $\left[\tilde{s}_{\alpha}, 1\right]$.

Remark B1. An alternative argument, contained in an earlier working paper of this study, analyzed instead the solution of the differential equation $q(b)=\psi(b, q(b))$ where

$$
\psi(b, q(b))=\frac{1-\sum_{k=0}^{N-1} \gamma_{k}^{\prime}(b) z_{k}(q(b) \mid q(b))}{\sum_{k=0}^{N-1} \gamma_{k}(b) z_{k}^{\prime}(q(b) \mid q(b))}, \quad q(\underline{w})=\tilde{s}_{\alpha}
$$

$b(s)$ is then defined as the inverse of $q(b)$. This approach assuages concerns related to the set of points where $\xi(s, b \mid s)=0$. For a similar approach, see Lebrun (1999) or Maskin and Riley (2003), among others.

Proof of Theorem 1. Let $U_{i}(b(x) \mid s, w)$ be the expected utility of bidder $i$ when she observes the value-signal $s$ and places the bid $b(x) \leq w$. Noting the definition of $b(x)$, it is 


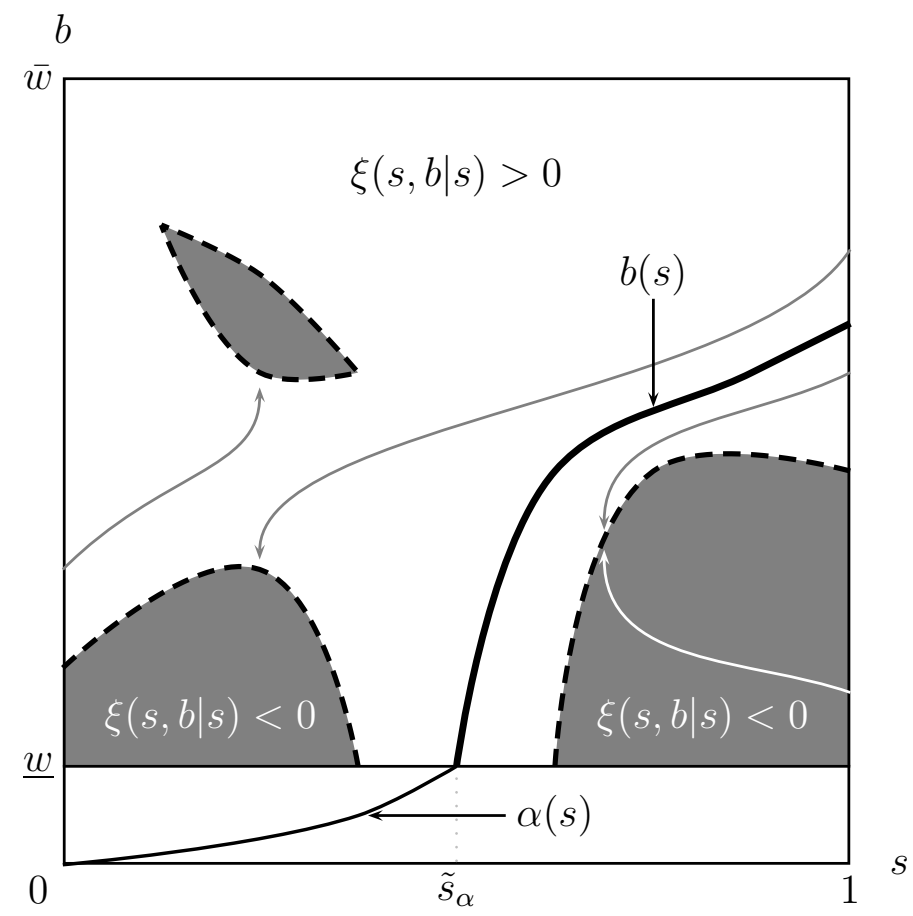

Figure B1: Definition of $b(s)$ in the all-pay auction. $b(s)$ is confined to the white region where all solutions to the differential equation (B1) are strictly increasing.

easily seen to be absolutely continuous. Moreover, since $G(\cdot)$ is continuously differentiable, $U_{i}(b(x) \mid s, w)=\sum_{k=0}^{N-1} \gamma_{k}(b(x)) z_{k}(x \mid s)-b(x)$ is absolutely continuous and in particular we can write

$$
U_{i}(b(x) \mid s, w)=U_{i}\left(b\left(\tilde{s}_{\alpha}\right) \mid s, w\right)+\left.\int_{\tilde{s}_{\alpha}}^{x} \frac{d}{d t} U_{i}(b(t) \mid s, w)\right|_{t=y} d y .
$$

We proceed to verify that no type of bidder wishes to deviate to an alternative (feasible) bid.

1. Consider a bidder with value-signal $s<\tilde{s}_{\alpha}$. When following the strategy $\beta(s, w)$, this bidder places the bid $b(s)=\alpha(s)$. From Krishna and Morgan (1997), we know that this bidder will not have a profitable deviation to any bid $b(x), x \in\left[0, \tilde{s}_{\alpha}\right]$.

Suppose instead that this bidder contemplates bidding $b(x)$ for some $x>\tilde{s}_{\alpha}$. The expected payoff from this bid is given by (B2). It is sufficient to verify that when 
$s<x, \frac{d}{d t} U_{i}(b(t) \mid s, w) \leq 0$. Using Assumptions A-3 and A-4, we can see that

$$
\begin{aligned}
& \frac{d}{d t} U_{i}(b(t) \mid s, w) \\
& =\sum_{k=0}^{N-1} \gamma_{k}^{\prime}(b(t)) z_{k}(t \mid s) b^{\prime}(t)+\sum_{k=0}^{N-1} \gamma_{k}(b(t)) z_{k}^{\prime}(t \mid s)-b^{\prime}(t) \\
& =\sum_{k=0}^{N-1} \gamma_{k}(b(t)) z_{k}^{\prime}(t \mid s)-\sum_{k=0}^{N-1} \gamma_{k}(b(t)) z_{k}^{\prime}(t \mid t)\left[\frac{1-\sum_{k=0}^{N-1} \gamma_{k}^{\prime}(b(t)) z_{k}(t \mid s)}{1-\sum_{k=0}^{N-1} \gamma_{k}^{\prime}(b(t)) z_{k}(t \mid t)}\right] \\
& \leq \sum_{k=0}^{N-1} \gamma_{k}(b(t)) z_{k}^{\prime}(t \mid t)-\sum_{k=0}^{N-1} \gamma_{k}(b(t)) z_{k}^{\prime}(t \mid t)\left[\frac{1-\sum_{k=0}^{N-1} \gamma_{k}^{\prime}(b(t)) z_{k}(t \mid t)}{1-\sum_{k=0}^{N-1} \gamma_{k}^{\prime}(b(t)) z_{k}(t \mid t)}\right]=0 .
\end{aligned}
$$

2. Consider instead a bidder with a value-signal $s \geq \tilde{s}_{\alpha}$. An argument parallel to the preceding case confirms that a bid $b(x), x>s$, will not be a profitable deviation. Suppose instead this bidder bids $b(x) \leq w, x \in\left[\tilde{s}_{\alpha}, s\right)$. As above, we have

$$
\frac{d}{d t} U_{i}(b(t) \mid s, w)=\sum_{k=0}^{N-1} \gamma_{k}(b(t)) z_{k}^{\prime}(t \mid s)-\sum_{k=0}^{N-1} \gamma_{k}(b(t)) z_{k}^{\prime}(t \mid t)\left[\frac{1-\sum_{k=0}^{N-1} \gamma_{k}^{\prime}(b(t)) z_{k}(t \mid s)}{1-\sum_{k=0}^{N-1} \gamma_{k}^{\prime}(b(t)) z_{k}(t \mid t)}\right]
$$

By Assumption A-3, $\sum_{k=0}^{N-1} \gamma_{k}(b(t)) z_{k}^{\prime}(t \mid s) \geq \sum_{k=0}^{N-1} \gamma_{k}(b(t)) z_{k}^{\prime}(t \mid t)$. There are two cases:

(a) If $1-\sum_{k=0}^{N-1} \gamma_{k}^{\prime}(b(t)) z_{k}(t \mid s)<0$, then $\frac{d}{d t} U_{i}(b(t) \mid s, w) \geq 0$.

(b) Suppose $1-\sum_{k=0}^{N-1} \gamma_{k}^{\prime}(b(t)) z_{k}(t \mid s) \geq 0$. Then by Assumption A-4,

$$
1-\sum_{k=0}^{N-1} \gamma_{k}^{\prime}(b(t)) z_{k}(t \mid s) \leq 1-\sum_{k=0}^{N-1} \gamma_{k}^{\prime}(b(t)) z_{k}(t \mid t)
$$

Hence, $\frac{d}{d t} U_{i}(b(t) \mid s, w) \geq 0$.

Therefore, there is no profitable deviation to a bid $b(x)$ when $x \in\left[\tilde{s}_{\alpha}, s\right)$.

Finally, consider a deviation to a bid of $b(x), x<\tilde{s}_{\alpha}$. It is sufficient to show that $\frac{d}{d t} U_{i}(b(t) \mid s, w) \geq 0$ for all $t<\tilde{s}_{\alpha}$. By Assumption A-3

$$
\begin{aligned}
\frac{d}{d t} U_{i}(b(t) \mid s, w) & =v_{N-1}(s, t) f_{N-1}(t \mid s)-v_{N-1}(t, t) f_{N-1}(t \mid t) \\
& \geq v_{N-1}(t, t) f_{N-1}(t \mid t)-v_{N-1}(t, t) f_{N-1}(t \mid t)=0
\end{aligned}
$$


The above analysis is exhaustive of all the cases; thus, the considered strategy profile is a symmetric equilibrium.

Proof of Corollary 1. By Assumption A-4, $1-\sum_{k=0}^{N-1} \gamma_{k}^{\prime}(\underline{w}) z_{k}\left(\tilde{s}_{\alpha} \mid \tilde{s}_{\alpha}\right)>0$. However, from Lemmas B1 and B2, $\sum_{k=0}^{N-1} \gamma_{k}^{\prime}(\underline{w}) z_{k}\left(\tilde{s}_{\alpha} \mid \tilde{s}_{\alpha}\right)>0$. Therefore,

$$
0<1-\sum_{k=0}^{N-1} \gamma_{k}^{\prime}(\underline{w}) z_{k}\left(\tilde{s}_{\alpha} \mid \tilde{s}_{\alpha}\right)<1
$$

Also, $\sum_{k=0}^{N-1} \gamma_{k}(b(s)) z_{k}^{\prime}(s \mid s)=\sum_{k=1}^{N-1} \gamma_{k}(b(s)) v_{k}(s, s) f_{k}(s \mid s)$. Since $\gamma_{k}(\underline{w})=0$ for all $k \neq N-1$ and $\gamma_{N-1}(\underline{w})=1$, we can take limits to conclude

$$
\begin{aligned}
\lim _{s \rightarrow \tilde{s}_{\alpha}^{+}} b^{\prime}(s) & =\lim _{s \rightarrow \tilde{s}_{\alpha}^{+}} \frac{\sum_{k=0}^{N-1} \gamma_{k}(b(s)) z_{k}^{\prime}(s \mid s)}{1-\sum_{k=0}^{N-1} \gamma_{k}^{\prime}(b(s)) z_{k}(s \mid s)} \\
& =\frac{\sum_{k=1}^{N-1} \gamma_{k}(\underline{w}) v_{k}\left(\tilde{s}_{\alpha}, \tilde{s}_{\alpha}\right) f_{k}\left(\tilde{s}_{\alpha} \mid \tilde{s}_{\alpha}\right)}{1-\sum_{k=0}^{N-1} \gamma_{k}^{\prime}(\underline{w}) z_{k}\left(\tilde{s}_{\alpha} \mid \tilde{s}_{\alpha}\right)} \\
& =\frac{v_{N-1}\left(\tilde{s}_{\alpha}, \tilde{s}_{\alpha}\right) f_{N-1}\left(\tilde{s}_{\alpha} \mid \tilde{s}_{\alpha}\right)}{1-\sum_{k=0}^{N-1} \gamma_{k}^{\prime}(\underline{w}) z_{k}\left(\tilde{s}_{\alpha} \mid \tilde{s}_{\alpha}\right)} \\
& >v_{N-1}\left(\tilde{s}_{\alpha}, \tilde{s}_{\alpha}\right) f_{N-1}\left(\tilde{s}_{\alpha} \mid \tilde{s}_{\alpha}\right)=\lim _{s \rightarrow \tilde{s}_{\alpha}^{-}} b^{\prime}(s) .
\end{aligned}
$$

Lemma B4. Consider an all-pay auction where the environment meets the conditions of Theorem 1 and $N=2$. Let $\beta(s, w)$ be a corresponding equilibrium strategy. Suppose $\alpha(s)$ is defined as in (9). Then $\beta(s, w) \leq \bar{\alpha}$.

Proof. It is sufficient to show that $b(s) \leq \bar{\alpha}$. Let $U_{\beta}(s)=\sum_{k=0}^{N-1} \gamma_{k}(b(s)) z_{k}(s \mid s)-b(s)$ be the equilibrium expected utility of a bidder placing the bid $b(s)$. For $k \geq 1$,

$$
\frac{d}{d s} z_{k}(s \mid s)=v_{k}(s, s) f_{k}(s \mid s)+\int_{0}^{s} \frac{\partial}{\partial s} v_{k}(s, y) f_{k}(y \mid s) d z=v_{k}(s, s) f_{k}(s \mid s)+\left.\frac{\partial}{\partial x} z_{k}(s \mid x)\right|_{x=s} .
$$

While for $k=0$,

$$
\frac{d}{d s} z_{0}(s \mid s)=\left.\frac{\partial}{\partial x} z_{0}(s \mid x)\right|_{x=s}
$$


Thus, for all $s>\tilde{s}_{\alpha}$ we can write

$$
U_{\beta}^{\prime}(s)=\left.\sum_{k=0}^{N-1} \gamma_{k}(b(s)) \frac{\partial}{\partial x} z_{k}(s \mid x)\right|_{x=s}
$$

We have cancelled terms using the definition of $b^{\prime}(s)$ from (7).

Similarly, let $\alpha(s)=\int_{0}^{s} v_{N-1}(y, y) f_{N-1}(y \mid y) d y$ and $U_{\alpha}(s)=\int_{0}^{s} v(s, y) f_{N-1}(y \mid s) d y-\alpha(s)$. By an analogous argument to that presented immediately above, we can show that

$$
U_{\alpha}^{\prime}(s)=\left.\frac{\partial}{\partial x} z_{N-1}(s \mid x)\right|_{x=s} .
$$

Therefore,

$$
U_{\beta}^{\prime}(s)-U_{\alpha}^{\prime}(s)=\sum_{k=0}^{N-1} \gamma_{k}(b(s))\left[\left.\frac{\partial}{\partial x} z_{k}(s \mid x)\right|_{x=s}-\left.\frac{\partial}{\partial x} z_{N-1}(s \mid x)\right|_{x=s}\right] .
$$

Before completing our argument, we verify one helpful fact: $z_{0}(s \mid \cdot)-z_{1}(s \mid \cdot):[0,1] \rightarrow \mathbb{R}$ is non-decreasing. To see this conclusion, note that for all $x$,

$$
\begin{aligned}
z_{0}(s \mid x)-z_{1}(s \mid x) & =\int_{s}^{1} u(x, y) h(y \mid x) d y \\
& =\operatorname{Pr}\left[Y_{1} \geq s \mid S=x\right] \mathbb{E}\left[u\left(x, Y_{1}\right) \mid Y_{1} \geq s, S=x\right] .
\end{aligned}
$$

Since, value-signals are affiliated and $u\left(x, y_{1}\right)$ is non-decreasing, $\operatorname{Pr}\left[Y_{1} \geq s \mid S=x\right]$ and $\mathbb{E}\left[u\left(x, Y_{1}\right) \mid Y_{1} \geq s, S=x\right]$ are non-decreasing in $x$.

Since $z_{0}(s \mid \cdot)-z_{1}(s \mid \cdot)$ is non-decreasing, for a.e. $s \geq \tilde{s}_{\alpha}, U_{\beta}^{\prime}(s) \geq U_{\alpha}^{\prime}(s)$. But then, since $U_{\beta}\left(\tilde{s}_{\alpha}\right)=U_{\alpha}\left(\tilde{s}_{\alpha}\right)$,

$$
U_{\beta}(s)=U_{\beta}\left(\tilde{s}_{\alpha}\right)+\int_{\tilde{s}_{\alpha}}^{s} U_{\beta}^{\prime}(x) d x \geq U_{\alpha}\left(\tilde{s}_{\alpha}\right)+\int_{\tilde{s}_{\alpha}}^{s} U_{\alpha}^{\prime}(x) d x=U_{\alpha}(s) .
$$

Taking $s \rightarrow 1$ and noting that $z_{k}(1 \mid 1)=z_{N-1}(1 \mid 1)$ for all $k$,

$$
U_{\beta}(1)=\sum_{k=0}^{N-1} \gamma_{k}(b(1)) z_{k}(1 \mid 1)-b(1)=z_{N-1}(1 \mid 1)-b(1) \geq U_{\alpha}(1)=z_{N-1}(1 \mid 1)-\alpha(1) .
$$

Thus, $\bar{\alpha}=\alpha(1) \geq b(1)$. Since $b(s)$ is non-decreasing, we can conclude that $\beta(s, w) \leq b(s) \leq$ $\bar{\alpha}$. 
Lemma B5 is used in the proof of Theorems 2 and 4.

Lemma B5. Suppose $(X, Y, Z)$ are affiliated random variables with a strictly positive, bounded, continuous density $f(x, y, z)$ defined on $[0,1]^{3}$. Define

$$
f(x \mid y, z)=\frac{f(x, y, z)}{\int_{0}^{1} f(x, y, z) d x} .
$$

Let $z^{\prime}>z$ and suppose that $f(\cdot \mid y, z) \neq f\left(\cdot \mid y, z^{\prime}\right)$ for all $y$. Then there exists $0<\hat{y}<1$ such that:

(a) $f\left(x \mid x, z^{\prime}\right)<f(x \mid x, z)$ for all $x<\hat{y}$.

(b) $\frac{f\left(x \mid x, z^{\prime}\right)}{1-F\left(x \mid x, z^{\prime}\right)}<\frac{f(x \mid x, z)}{1-F(x \mid x, z)}$ for all $x<\hat{y}$.

Proof. Let $z^{\prime}>z$ and fix $y$. Since $\left(y, z^{\prime}\right) \geq(y, z)$, by the properties of affiliated random variables the function

$$
\frac{f\left(\cdot \mid y, z^{\prime}\right)}{f(\cdot \mid y, z)}:[0,1] \rightarrow \mathbb{R}
$$

is non-decreasing. It is also continuous and strictly positive.

Suppose $f\left(0 \mid y, z^{\prime}\right) \geq f(0 \mid y, z)$. Then $f\left(x \mid y, z^{\prime}\right) \geq f(x \mid y, z)$ for all $x \in[0,1]$. Since $f(\cdot \mid y, z) \neq f\left(\cdot \mid y, z^{\prime}\right)$ there exist an open set $\mathcal{X} \subset[0,1]$ such that for all $x \in \mathcal{X}, f(x \mid y, z)>$ $f\left(x \mid y, z^{\prime}\right)$. But this implies $1=\int_{0}^{1} f(x \mid y, z) d x<\int_{0}^{1} f\left(x \mid y, z^{\prime}\right) d x$ which is a contradiction. Therefore $f\left(0 \mid y, z^{\prime}\right)<f(0 \mid y, z)$. Specifically, the above conclusion holds when $y=0$ : $f\left(0 \mid 0, z^{\prime}\right)<f(0 \mid 0, z)$. Noting that $f\left(\cdot \mid \cdot, z^{\prime}\right)$ and $f(\cdot \mid \cdot, z)$ are continuous functions, there exists $\hat{y}>0$ such that for all $0<x<\hat{y}, f\left(x \mid x, z^{\prime}\right)<f(x \mid x, z)$ as desired.

To derive the second conclusion, let $x<\hat{y}$. Then for all $\tilde{x} \leq x$,

$$
1 \geq \frac{f\left(x \mid x, z^{\prime}\right)}{f(x \mid x, z)} \geq \frac{f\left(\tilde{x} \mid x, z^{\prime}\right)}{f(\tilde{x} \mid x, z)}
$$

Thus, $F\left(x \mid x, z^{\prime}\right)=\int_{0}^{x} f\left(\tilde{x} \mid x, z^{\prime}\right) d \tilde{x} \leq \int_{0}^{x} f(\tilde{x} \mid x, z) d \tilde{x}=F(x \mid x, z)$. Hence,

$$
\frac{1}{1-F\left(x \mid x, z^{\prime}\right)} \leq \frac{1}{1-F(x \mid x, z)}
$$

Combining this observation with the first conclusion gives the second result.

Proof of Theorem 2. Let $\bar{\beta}(s, w)=\min \{\bar{b}(s), w\}$ and $\underline{\beta}(s, w)=\min \{\underline{b}(s), w\}$ be the equilibrium bidding strategies conditional on the realized public signal. To prove part (1) it 
is sufficient to verify that $\bar{b}(s) \geq \underline{b}(s)$. Since the value-signal is only value-relevant we can let $\bar{u}(\underline{u})$ be a bidder's utility function when the public signals is high (low). The values $\bar{z}_{k}$, $\bar{v}_{k}, \underline{z}_{k}$, and $\underline{v}_{k}$ are defined in the obvious way. Since $\bar{u}>\underline{u}$ a.e., it follows that $\bar{v}_{k}>\underline{v}_{k}$ and $\bar{z}_{k}>\underline{z}_{k}$. Moreover, $\bar{z}_{k}-\bar{z}_{k+1} \geq \underline{z}_{k}-\underline{z}_{k+1}$, and thus $\sum_{k=0}^{N-1} \gamma_{k}^{\prime}(w) \bar{z}_{k}(s \mid s) \geq \sum_{k=0}^{N-1} \gamma_{k}^{\prime}(w) \underline{z}_{k}(s \mid s)$.

Thus, when $\bar{b}(s) \leq \underline{w}$, we have

$$
\bar{\alpha}(s)=\bar{b}(s)=\int_{0}^{s} \bar{v}_{N-1}(y, y) f_{k}(y \mid y) d y>\int_{0}^{s} \underline{v}_{N-1}(y, y) f_{k}(y \mid y) d y=\underline{b}(s)=\underline{\alpha}(s) .
$$

Thus, if $\bar{\alpha}\left(\tilde{\bar{s}}_{\alpha}\right)=\underline{w}$, there exists $s^{*}>\tilde{\bar{s}}_{\alpha}$ such that for all $s \leq s^{*}, \bar{b}(s) \geq \underline{b}(s)$. Suppose that at $s^{*}, \bar{b}\left(s^{*}\right)=\underline{b}\left(s^{*}\right)=b^{*}$ and for all $\epsilon>0$ sufficiently small, $\bar{b}\left(s^{*}+\epsilon\right)<\underline{b}\left(s^{*}\right)$. Since $\bar{b}^{\prime}(s)$ and $\underline{b}^{\prime}(s)$ are both continuous functions, we see that

$$
\begin{aligned}
\underline{b}^{\prime}\left(s^{*}\right) & =\frac{\sum_{k=1}^{N-1} \gamma_{k}\left(b^{*}\right) \underline{v}_{k}\left(s^{*}, s^{*}\right) f_{k}\left(s^{*} \mid s^{*}\right)}{1-\sum_{k=0}^{N-1} \gamma_{k}^{\prime}\left(b^{*}\right) \underline{z}_{k}\left(s^{*} \mid s^{*}\right)} \\
& <\frac{\sum_{k=1}^{N-1} \gamma_{k}\left(b^{*}\right) \bar{v}_{k}\left(s^{*}, s^{*}\right) f_{k}\left(s^{*} \mid s^{*}\right)}{1-\sum_{k=0}^{N-1} \gamma_{k}^{\prime}\left(b^{*}\right) \bar{z}_{k}\left(s^{*} \mid s^{*}\right)}=\bar{b}^{\prime}\left(s^{*}\right),
\end{aligned}
$$

implying a contradiction. Therefore $\bar{b}(s) \geq \underline{b}(s)$ for all $s$.

Turning to part (2), we know that $b(s)=\int_{0}^{s} v_{N-1}(y, y) f_{N-1}\left(y \mid y, s_{0}\right) d y$ for all $s>0$ sufficiently small. Lemma B5 implies that if $\bar{s}_{0}>\underline{s}_{0}$, then $f_{N-1}\left(y \mid y, \bar{s}_{0}\right)<f_{N-1}\left(y \mid y, \underline{s}_{0}\right)$ for all $0<y$ sufficiently small. Therefore, $\underline{b}(s) \geq \bar{b}(s)$ for all $s>0$ sufficiently small.

Lemmas B6 and B7 are used to prove Theorem 3.

Lemma B6. Let

$$
F_{k}(x \mid s)=\underbrace{\int_{0}^{1} \cdots \int_{0}^{1}}_{N-1-k} \underbrace{\int_{0}^{x} \cdots \int_{0}^{x}}_{k} h\left(y_{1}, \ldots, y_{N-1} \mid s\right) d y_{1} \cdots d y_{N-1}
$$

and let $f_{k}(x \mid s)=\frac{d}{d x} F_{k}(x \mid s)$ be the associated density. Then for all $k \geq 1, \frac{f_{k}(s \mid s)}{1-F_{k}(s \mid s)} \geq$ $\left(\frac{k}{k+1}\right) \frac{f_{k+1}(s \mid s)}{1-F_{k+1}(s \mid s)}$.

Proof. We note that $F_{k}(x \mid s) \geq F_{k+1}(x \mid s)$. Using the symmetry of $h(\cdot)$, we can compute 
$f_{k}(x \mid s)$ to conclude

$$
\begin{aligned}
\frac{f_{k}(x \mid s)}{1-F_{k}(x \mid s)} & =\frac{k \overbrace{\int_{0}^{1} \ldots \int_{0}^{1}}^{N-1-k} \overbrace{\int_{0}^{x} \ldots \int_{0}^{x}}^{k-1} h\left(y_{1}, \ldots, y_{N-2}, x \mid s\right) d y_{1} \ldots d y_{N-2}}{1-F_{k}(x \mid s)} \\
& \geq \frac{\frac{k}{k+1}(k+1) \overbrace{\int_{0}^{1} \cdots \int_{0}^{1}}^{N-2-k} \overbrace{\int_{0}^{x} \ldots \int_{0}^{x}}^{k} h\left(y_{1}, \ldots, y_{N-2}, x \mid s\right) d y_{1} \ldots d y_{N-2}}{1-F_{k+1}(x \mid s)} \\
& =\left(\frac{k}{k+1}\right) \frac{f_{k+1}(x \mid s)}{1-F_{k+1}(x \mid s)} .
\end{aligned}
$$

Lemma B7. The differential equation

$$
b^{\prime}(s)=\frac{\sum_{k=0}^{N-1} \gamma_{k}(b(s)) z_{k}^{\prime}(s \mid s)}{1-\hat{H}(s, b(s) \mid s)-\sum_{k=0}^{N-1} \gamma_{k}^{\prime}(b(s)) z_{k}(s \mid s)}
$$

has a strictly increasing solution, $b(s)$, satisfying the boundary condition $b\left(\tilde{s}_{\omega}\right)=\underline{w}$. Moreover, there exists $\hat{s}_{\omega} \leq 1$ such that $\lim _{s \rightarrow \hat{s}_{\omega}^{-}} b(s)=\bar{w}$.

Proof. The existence of a strictly increasing solution follows from the same reasoning as presented in the case of the all-pay auction. In particular, Assumption A-6 ensures that $b^{\prime}(s)>0$ in the relevant range of values. We therefore focus on showing the final claim that $\hat{b}(s)$ tends to $\bar{w}$.

Applying Lemma B6 multiple times lets us conclude that

$$
\frac{f_{k}(x \mid s)}{1-F_{k}(x \mid s)} \geq\left(\frac{k}{N-1}\right) \frac{f_{N-1}(x \mid s)}{1-F_{N-1}(x \mid s)} \geq\left(\frac{1}{N-1}\right) \frac{f_{N-1}(x \mid s)}{1-F_{N-1}(x \mid s)} .
$$


Also, noting Lemma B1, $\sum_{k=0}^{N-1} \gamma_{k}^{\prime}(b(s)) z_{k}(s \mid s) \geq 0$. Thus,

$$
\begin{aligned}
b^{\prime}(s) & =\frac{\sum_{k=0}^{N-1} \gamma_{k}(b(s)) z_{k}^{\prime}(s \mid s)}{1-\hat{H}(s, b(s) \mid s)-\sum_{k=0}^{N-1} \gamma_{k}^{\prime}(b(s)) z_{k}(s \mid s)} \\
& \geq \frac{\sum_{k=0}^{N-1} \gamma_{k}(b(s)) z_{k}^{\prime}(s \mid s)}{1-\hat{H}(s, b(s) \mid s)} \\
& =\frac{\sum_{k=1}^{N-1} \gamma_{k}(b(s)) v_{k}(s, s) f_{k}(s \mid s)}{\sum_{k=1}^{N-1} \gamma(b(s))\left(1-F_{k}(s \mid s)\right)} \\
& \geq v_{N-1}(s, s) \frac{\sum_{k=1}^{N-1} \gamma_{k}(b(s)) f_{k}(s \mid s)}{\sum_{k=1}^{N-1} \gamma(b(s))\left(1-F_{k}(s \mid s)\right)} \\
& =v_{N-1}(s, s) \sum_{k=1}^{N-1} \frac{\gamma_{k}(b(s))\left(1-F_{k}(s \mid s)\right)}{\sum_{k=1}^{N-1} \gamma_{k}(b(s))\left(1-F_{k}(s \mid s)\right)} \frac{f_{k}(s \mid s)}{1-F_{k}(s \mid s)} \\
& \geq v_{N-1}(s, s) \sum_{k=1}^{N-1} \frac{\gamma_{k}(b(s))\left(1-F_{k}(s \mid s)\right)}{\sum_{k=1}^{N-1} \gamma_{k}(b(s))\left(1-F_{k}(s \mid s)\right)}\left(\frac{1}{N-1}\right) \frac{f_{N-1}(s \mid s)}{1-F_{N-1}(s \mid s)} \\
& =\frac{1}{N-1} \frac{v_{N-1}(s, s) f_{N-1}(s \mid s)}{1-F_{N-1}(s \mid s)}=\frac{\omega^{\prime}(s)}{N-1}
\end{aligned}
$$

Since $b\left(\tilde{s}_{\omega}\right)=\omega\left(\tilde{s}_{\omega}\right)=\underline{w}$, we have that for $s \geq \tilde{s}_{\omega}, b(s)=\underline{w}+\int_{\tilde{s}_{\omega}}^{s} b^{\prime}(x) d x \geq \underline{w}+$ $\frac{1}{N-1} \int_{\tilde{s}_{\omega}}^{s} \omega^{\prime}(x) d x=\underline{w}+\frac{1}{N-1}(\omega(s)-\underline{w})$. From Krishna and Morgan (1997, Proposition 1), $\lim _{s \rightarrow 1} \omega(s)=\infty$. Thus, for some $\hat{s}_{\omega} \leq 1, \lim _{s \rightarrow \hat{s}_{\omega}^{-}} \hat{b}(s)=\bar{w}$.

Proof of Theorem 3. Noting Lemma B7, here we only verify that the proposed strategy is an equilibrium. The argument proceeds similarly to the case of the all-pay auction. If $\beta(s, w)=\min \{b(s), w\}$ is the proposed equilibrium strategy, the range of $\beta$ equals the range of $b(s)$; therefore, we need to rule out deviations only to alternative bids $b(x)$ for some $x \leq \hat{s}_{\omega}$. As in the case of the all-pay auction, we can write the expected payoff from the bid $b(x)$ as

$$
U_{i}(b(x) \mid s, w)=U_{i}\left(b\left(\tilde{s}_{\omega}\right) \mid s, w\right)+\left.\int_{\tilde{s}_{\omega}}^{x} \frac{d}{d t} U(b(t) \mid s, w)\right|_{t=y} d y
$$

1. Consider a bidder with a value-signal $s<\tilde{s}_{\omega}$. When following the strategy $\beta(s, w)$, this bidder places the bid $b(s)=\omega(s)$. From Krishna and Morgan (1997), we known that this bidder will not have a profitable deviation to any bid $b(x), x \in\left[0, \tilde{s}_{\omega}\right]$.

Suppose instead that this bidder contemplates bidding $b(x)$ for some $x>\tilde{s}_{\omega}$. To confirm this alternative is inferior, it is sufficient to verify that $\frac{d}{d t} U_{i}(b(t) \mid s, w) \leq 0$ for 
a.e. $t \in\left[\tilde{s}_{\omega}, x\right]$. Differentiating and simplifying as needed we see that

$$
\begin{aligned}
& \frac{d}{d t} U_{i}(b(t) \mid s, w) \\
& =\sum_{k=1}^{N-1} \gamma_{k}(b(t)) v_{k}(s, t) f_{k}(t \mid s)-b^{\prime}(t)\left(\sum_{k=1}^{N-1} \gamma_{k}(b(t))\left(1-F_{k}(t \mid s)\right)-\sum_{k=0}^{N-1} \gamma_{k}^{\prime}(b(t)) z_{k}(t \mid s)\right) \\
& =\left[\sum_{k=1}^{N-1} \gamma_{k}(b(t))\left(1-F_{k}(t \mid s)\right)\right]\left(\Phi(t, b(t) \mid s)-b^{\prime}(t) \Xi(t, b(t) \mid s)\right) \\
& \leq\left[\sum_{k=1}^{N-1} \gamma_{k}(b(t))\left(1-F_{k}(t \mid s)\right)\right]\left(\Phi(t, b(t) \mid t)-b^{\prime}(t) \Xi(t, b(t) \mid t)\right)=0
\end{aligned}
$$

The inequality follows from Assumptions A-5 and A-6. The final equality is due to the observation that for $t>\tilde{s}_{\omega}, b^{\prime}(t)=\Phi(t, b(t) \mid t) / \Xi(t, b(t) \mid t)$. Thus, the bid $b(x), x>\tilde{s}_{\omega}$ is not a profitable deviation.

2. Consider a bidder with a value-signal $s>\tilde{s}_{\alpha}$. The preceding argument continues to apply and this bidder will not have a profitable deviation to any bid $b(x) \leq w$ such that $x \geq s$. Suppose instead $x \in\left[\tilde{s}_{\omega}, s\right]$. To rule out such a deviation, it is sufficient to show that $\frac{d}{d t} U_{i}(b(t) \mid s, w) \geq 0$ for a.e. $t \in[x, s]$. By Assumption A-5, we know that

$$
\begin{aligned}
\frac{d}{d t} U_{i}(b(t) \mid s, w) & =\left[\sum_{k=1}^{N-1} \gamma_{k}(b(t))\left(1-F_{k}(t \mid s)\right)\right]\left(\Phi(t, b(t) \mid s)-b^{\prime}(t) \Xi(t, b(t) \mid s)\right) \\
& \geq\left[\sum_{k=1}^{N-1} \gamma_{k}(b(t))\left(1-F_{k}(t \mid s)\right)\right]\left(\Phi(t, b(t) \mid t)-b^{\prime}(t) \Xi(t, b(t) \mid s)\right)
\end{aligned}
$$

If $\Xi(t, b(t) \mid s) \leq 0$, then $\frac{d}{d t} U_{i}(b(t) \mid s, w) \geq 0$. If instead $\Xi(t, b(t) \mid s) \geq 0$, then from Assumption A-6, $\Xi(t, b(t) \mid s) \leq \Xi(t, b(t) \mid t)$. Thus, $\frac{d}{d t} U_{i}(b(t) \mid s, w) \geq 0$ as required.

Finally, consider a deviation to a bid $b(x), x \leq \tilde{s}_{\omega}$. It is sufficient to show that $\frac{d}{d t} U_{i}(b(t) \mid s, w) \geq 0$ for $t \in\left[x, \tilde{s}_{\omega}\right]$. Differentiating and simplifying gives

$$
\begin{aligned}
\frac{d}{d t} U_{i}(b(t) \mid s, w) & =v_{N-1}(s, t) \frac{f_{N-1}(t \mid s)}{1-F_{N-1}(t \mid s)}-v_{N-1}(t, t) \frac{f_{N-1}(t \mid t)}{1-F_{N-1}(t \mid t)} \\
& \geq v_{N-1}(t, t) \frac{f_{N-1}(t \mid t)}{1-F_{N-1}(t \mid t)}-v_{N-1}(t, t) \frac{f_{N-1}(t \mid t)}{1-F_{N-1}(t \mid t)}=0
\end{aligned}
$$

Since the above analysis is exhaustive of all possible cases, we conclude that $\beta(s, w)$ is an 
equilibrium.

Proof of Corollary 2. The proof of part (1) is analogous to the proof of Corollary 1. Additionally, we note that $\hat{H}\left(\tilde{s}_{\omega}, \underline{w} \mid \tilde{s}_{\omega}\right)=F_{N-1}\left(\tilde{s}_{\omega} \mid \tilde{s}_{\omega}\right)$. Thus,

$$
\begin{aligned}
\lim _{s \rightarrow \tilde{s}_{\omega}^{+}} b^{\prime}(s) & =\frac{v_{N-1}\left(\tilde{s}_{\omega}, \tilde{s}_{\omega}\right) f_{N-1}\left(\tilde{s}_{\omega} \mid \tilde{s}_{\omega}\right)}{1-F_{N-1}\left(\tilde{s}_{\omega} \mid \tilde{s}_{\omega}\right)-\sum_{k=0}^{N-1} \gamma_{k}^{\prime}(\underline{w}) z_{k}\left(\tilde{s}_{\omega} \mid \tilde{s}_{\omega}\right)} \\
& >\frac{v_{N-1}\left(\tilde{s}_{\omega}, \tilde{s}_{\omega}\right) f_{N-1}\left(\tilde{s}_{\omega} \mid \tilde{s}_{\omega}\right)}{1-F_{N-1}\left(\tilde{s}_{\omega} \mid \tilde{s}_{\omega}\right)} \\
& =\lim _{s \rightarrow \tilde{s}_{\omega}^{-}} b^{\prime}(s) .
\end{aligned}
$$

For part (2) it is sufficient to verify that $b^{\prime}(s) \geq \omega^{\prime}(s)$.

$$
\begin{aligned}
b^{\prime}(s) & =\frac{\sum_{k=0}^{N-1} \gamma_{k}(b(s)) z_{k}^{\prime}(s \mid s)}{1-\hat{H}(s, b(s) \mid s)-\sum_{k=0}^{N-1} \gamma_{k}^{\prime}(b(s)) z_{k}(s \mid s)} \\
& =\frac{\sum_{k=1}^{N-1}\left[\frac{\gamma_{k}(b(s))\left(1-F_{k}(s \mid s)\right)}{\sum_{k^{\prime}=1}^{N-1} \gamma_{k^{\prime}}(b(s))\left(1-F_{k^{\prime}}(s \mid s)\right)}\right] \frac{v_{k}(s, s) f_{k}(s \mid s)}{1-F_{k}(s \mid s)}}{1-\frac{\sum_{k=0}^{N-1} \gamma_{k}^{\prime}(b(s)) z_{k}(s \mid s)}{\sum_{k^{\prime}=1}^{N-1} \gamma_{k^{\prime}}(b(s))\left(1-F_{k^{\prime}}(s \mid s)\right)}} \\
& \geq \sum_{k=1}^{N-1}\left[\frac{\gamma_{k}(b(s))\left(1-F_{k}(s \mid s)\right)}{\sum_{k^{\prime}=1}^{N-1} \gamma_{k^{\prime}}(b(s))\left(1-F_{k^{\prime}}(s \mid s)\right)}\right] \frac{v_{k}(s, s) f_{k}(s \mid s)}{1-F_{k}(s \mid s)} \\
& \geq \frac{v_{N-1}(s, s) f_{N-1}(s \mid s)}{1-F_{N-1}(s \mid s)}=\omega^{\prime}(s) .
\end{aligned}
$$

Proof of Theorem 4. The argument is analogous to the proof of Theorem 2. It also calls upon Lemma B5 where part (b) addresses the case of interest.

Proof of Theorem 5. Since $v_{N-1}(s, s) f_{N-1}(s \mid s)<v_{N-1}(s, s) \frac{f_{N-1}(s \mid s)}{1-F_{N-1}(s \mid s)}, b_{\omega}(s)>b_{\alpha}(s)$ for all $s \in\left(0, \tilde{s}_{\alpha}\right]$. Noting the proof of Theorem 2 it is sufficient to verify that if there exists a $\left(s^{*}, b^{*}\right)$ such that $b^{*}=b_{\omega}\left(s^{*}\right)=b_{\alpha}\left(s^{*}\right)$, then $b_{\omega}^{\prime}\left(s^{*}\right)>b_{\alpha}^{\prime}\left(s^{*}\right)$ which will imply a contradiction. 
This however is straightforward since

$$
\begin{aligned}
b_{\omega}^{\prime}\left(s^{*}\right) & =\frac{\sum_{k=0}^{N-1} \gamma_{k}\left(b^{*}\right) z_{k}^{\prime}\left(s^{*} \mid s^{*}\right)}{1-\hat{H}\left(s^{*}, b^{*} \mid s^{*}\right)-\sum_{k=0}^{N-1} \gamma_{k}^{\prime}\left(b^{*}\right) z_{k}\left(s^{*} \mid s^{*}\right)} \\
& >\frac{\sum_{k=0}^{N-1} \gamma_{k}\left(b^{*}\right) z_{k}^{\prime}\left(s^{*} \mid s^{*}\right)}{1-\sum_{k=0}^{N-1} \gamma_{k}^{\prime}\left(b^{*}\right) z_{k}\left(s^{*} \mid s^{*}\right)}=b_{\alpha}^{\prime}\left(s^{*}\right) .
\end{aligned}
$$




\section{References}

Albano, G. L. (2001). A class of all-pay auctions with affiliated information. Recherches Économiques de Louvain, 67(1):31-38.

Araujo, A., de Castro, L. I., and Moreira, H. (2008). Non-monotinicities and the all-pay auction tie-breaking rule. Economic Theory, 35(3):407-440.

Athey, S. and Haile, P. A. (2007). Nonparametric approaches to auctions. In Handbook of Econometrics, volume 6A, chapter 60, pages 3847-3965. Elsevier Science Publishers.

Avery, C., Fairbanks, A., and Zeckhauser, R. J. (2004). The Early Admissions Game: Joining the Elite. Harvard University Press, Cambridge, MA.

Bajari, P. and Hortaçsu, A. (2005). Are structural estimates of auction models reasonable? Evidence from experimental data. Journal of Political Economy, 113(4):703-741.

Baye, M. R., Kovenock, D., and de Vries, C. G. (1993). Rigging the lobbying process: An application of the all-pay auction. American Economic Review, 83(1):289-294.

Baye, M. R., Kovenock, D., and de Vries, C. G. (2005). Comparative analysis of litigation systems: An auction-theoretic approach. The Economic Journal, 115(505):583-601.

Che, Y.-K. and Gale, I. (1996). Expected revenue of all-pay auctions and first price sealed-bid auctions with budget constraints. Economics Letters, 50(3):373-379.

Che, Y.-K. and Gale, I. (1998a). Caps on political lobbying. American Economic Review, 88(3):643-651.

Che, Y.-K. and Gale, I. (1998b). Standard auctions with financially constrained bidders. Review of Economic Studies, 65(1):1-21.

Che, Y.-K. and Gale, I. (2006). Revenue comparisons for auctions when bidders have arbitrary types. Theoretical Economics, 1(1):95-118.

Corazzini, L., Faravelli, M., and Stanca, L. (2010). A prize to give for: An experiment on public good funding mechanisms. The Economic Journal, 120(547):944-967.

de Castro, L. I. (2010). Affiliation, equilibrium existence and revenue ranking of auctions. Mimeo.

Dechenaux, E., Kovenock, D., and Sheremeta, R. M. (2012). A survey of experimental research on contests, all-pay auctions and tournaments. Mimeo.

Dekel, E., Jackson, M. O., and Wolinsky, A. (2006). Jump bidding and budget constraints in all-pay auctions and wars of attrition. Mimeo. 
Fang, H. and Parreiras, S. (2002). Equilibrium of affiliated value second price auctions with financially constrained bidders: The two-bidder case. Games and Economic Behavior, $39(2): 215-236$.

Fang, H. and Parreiras, S. (2003). On the failure of the linkage principle with financially constrained bidders. Journal of Economic Theory, 110(2):374-392.

Gavious, A., Moldovanu, B., and Sela, A. (2002). Bid costs and endogenous bid caps. RAND Journal of Economics, 33(4):709-722.

Goeree, J. K., Maasland, E., Onderstal, S., and Turner, J. L. (2005). How (not) to raise money. Journal of Political Economy, 113(4):897-918.

Hickman, B. R. (2011). Effort, race gaps and affirmative action: A game-theoretic analysis of college admissions. Mimeo.

Hillman, A. L. and Riley, J. G. (1989). Politically contestable rents and transfers. Economics and Politics, 1(1):17-39.

Hörisch, H. and Kirchkamp, O. (2010). Less fighting than expected: Experiments with wars of attrition and all-pay auctions. Public Choice, 144(1-2):347-367.

Konrad, K. A. (2009). Strategy and Dynamics in Contests. Oxford University Press, Oxford.

Kotowski, M. H. (2010). All-pay auctions with budget constraints. Mimeo.

Kotowski, M. H. (2013). First-price auctions with budget constraints. Mimeo.

Kotowski, M. H. and Li, F. (2012). On the continuous equilibria of affiliated-value, all-pay auctions with private budget constraints. Mimeo.

Krishna, V. (2002). Auction Theory. Academic Press, San Diego, CA.

Krishna, V. and Morgan, J. (1997). An analysis of the war of attrition and the all-pay auction. Journal of Economic Theory, 72(2):343-362.

Laffont, J.-J. and Robert, J. (1996). Optimal auction with financially constrained buyers. Economics Letters, 52(2):181-186.

Lebrun, B. (1999). First price auctions in the asymmetric $\mathrm{N}$ bidder case. International Economic Review, 40(1):125-142.

Leininger, W. (1991). Patent competition, rent dissipation, and the persistence of monopoly: The role of research budgets. Journal of Economic Theory, 53(1):146-172.

Li, F. (2010). Equilibrium of affliated value all-pay auctions with budget constraint: The two-bidder case. Mimeo. 
Maskin, E. S. (2000). Auctions, development, and privatization: Efficient auctions with liquidity-constrained buyers. European Economic Review, 44(4-6):667-681.

Maskin, E. S. and Riley, J. G. (2003). Uniqueness of equilibrium in sealed high-bid auctions. Games and Economic Behavior, 45(2):395-409.

Milgrom, P. R. and Weber, R. J. (1982). A theory of auctions and competitive bidding. Econometrica, 50(5):1089-1122.

Morgan, J. (2000). Financing public goods by means of lotteries. Review of Economic Studies, 67(4):761-784.

Pai, M. M. and Vohra, R. (Forthcoming). Optimal auctions with financially constrained buyers. Journal of Economic Theory.

Tullock, G. (1980). Efficient rent seeking. In Buchanan, J. M., Tollison, R. D., and Tullock, G., editors, Toward a Theory of the Rent-Seeking Society, pages 97-112. Texas A\&M University Press.

Zheng, C. Z. (2001). High bids and broke winners. Journal of Economic Theory, 100(1):129171. 